\title{
Coherent Optical Sources for Communications
}

\author{
JOSEPH E. GEUSIC, WILLIAM B. BRIDGES, FELLOW, IEEE, AND \\ JACQUES I. PANKOVE, FELLOW, IEEE
}

\begin{abstract}
The development of cohorent optical sources, producing usable amounts of power, has provided a stimulus for communications research. Coherent sources in the form of lasers and parametric oscillators are available at wavelengths which span the entire optical spectrum. This paper reviews the state of the art of coherent optical sources with major emphasis on the most highly developed sources.
\end{abstract}

\section{INTRODUCTION}

$\mathrm{T}$ THE introduction of the laser in 1960 and the subsequent work on intense coherent optical sources within the past decade has stimulated research in optical communications. An imposing number of coherent sources have been demonstrated with useful power outputs at wavelengths which span the optical spectrum. Lasers of all types (solid-state ion, semiconductor, liquid, and gaseous), both pulsed and continuous, have been utilized to investigate specific optical communication problems relating to modulation, detection, terminal design, and propagation. Since the discrete nature of laser transitions permits only limited frequency tuning of such sources, the laser has been combined with nonlinear optical materials to produce optical parametric oscillators with wide frequency tuning capabilities. These latter sources have not yet been used in optical communications research but are certain to play an important role in its future.

The purpose of this paper is to review those coherent sources and related techniques which are believed to be important to current and future optical communication research and system planning. In general the discussion will emphasize continuously operating sources at room temperature, since they are the most suitable for communication experiments and systems. Section II is a brief discussion of the principles of operation of coherent optical sources, and Section III covers representative methods of controlling the spatial and temporal behavior of optical oscillators. The state of the art of gas lasers, semiconductor lasers, solid-state ion lasers, and optical parametric oscillators is reviewed in Sections IV-VII.

\section{Types of Coherent Optical Sources}

A brief description of the principles of operation of the basic optical source types (lasers, parametric oscillators, and second-harmonic generators) is given in this section.

\section{A. Lasers}

The maser principle, which was originally developed in the microwave region, is the basis for the laser. Application

Manuscript received in final form August 21, 1970.

J. E. Geusic is with Bell Telephone Laboratories, Murray Hill, N. J. W. B. Bridges is with Hughes Research Laboratories, Malibu, Calif. J. I. Pankove is with RCA Laboratories, Princeton, N. J.

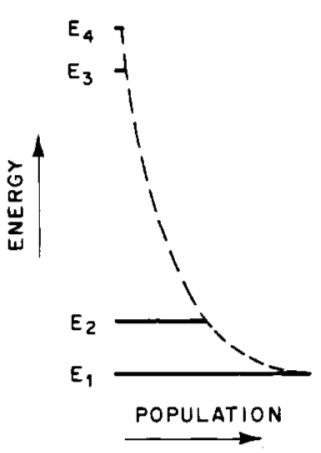

(a)

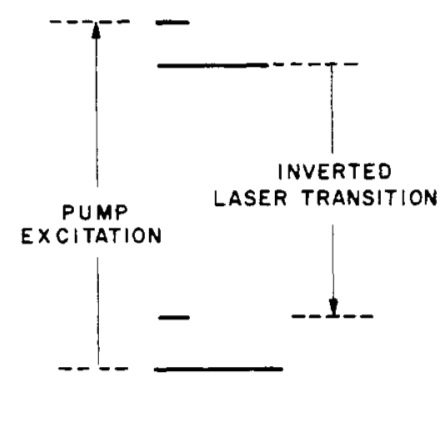

(b)
Fig. 1. Four-level system. (a) Without pumping. (b) With pumping excitation applied between levels $E_{1}$ and $E_{4}$ to produce inverted population between levels $E_{3}$ and $E_{2}$.

of this principle to optical transitions provided the first method of achieving net gain at optical frequencies. Extension of the maser principle to optics was first proposed by Schawlow and Townes in 1958 [1] and applied by Maiman in 1960 [2] to optical transitions in ruby to produce the pulsed ruby laser. Less than a year later Javan et al. [3] reported operation of the first continuous-wave (CW) laser, which used a $\mathrm{He}-\mathrm{Ne}$ discharge. Since then laser action has been demonstrated in numerous systems.

Strictly speaking, a laser is an amplifier of light. In all the lasers which will be discussed, optical gain is achieved by utilizing a medium which has three or more energy levels. The four-level system shown in Fig. 1 is illustrative. As depicted in Fig. 1(a), in the absence of pumping the population of the lower levels exceeds that of higher levels and all transitions are absorptive. If pumping excitation (the pumping may be optical, electrical, or chemical depending on the system) is applied selectively between levels $E_{1}$ and $E_{4}$, the level populations are modified. Under some conditions, for example, the case in which particles in levels $E_{4}$ and $E_{2}$ decay rapidly and predominantly to levels $E_{3}$ and $E_{1}$, respectively, the population of level $E_{3}$ will exceed that of $E_{2}$, as is shown in Fig. 1(b) and the system will exhibit optical gain at frequency $v_{32}$.

A laser oscillator is constructed by utilizing the laser gain medium inside of an optical cavity as is shown in Fig. 2(a). Optical regenerative gain occurs for light traveling along the cavity axis. The cavity length $l$ is typically $10^{3}$ to $10^{6}$ times larger than the laser wavelength, and typically more than one axial or longitudinal cavity resonance will fall within the laser gain profile. Oscillation occurs [4] at those cavity resonances lying within the inhomogeneous width of the laser transition for which the laser gain exceeds the cavity losses. This is depicted in Fig. 2(b) for a situation where the laser peak gain exceeds the single-pass cavity 


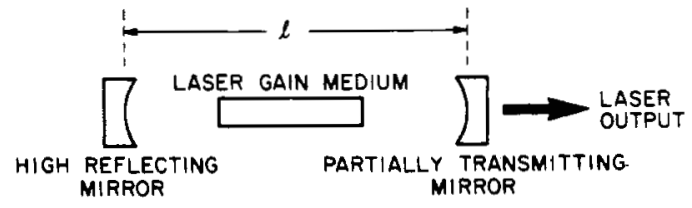

(a)
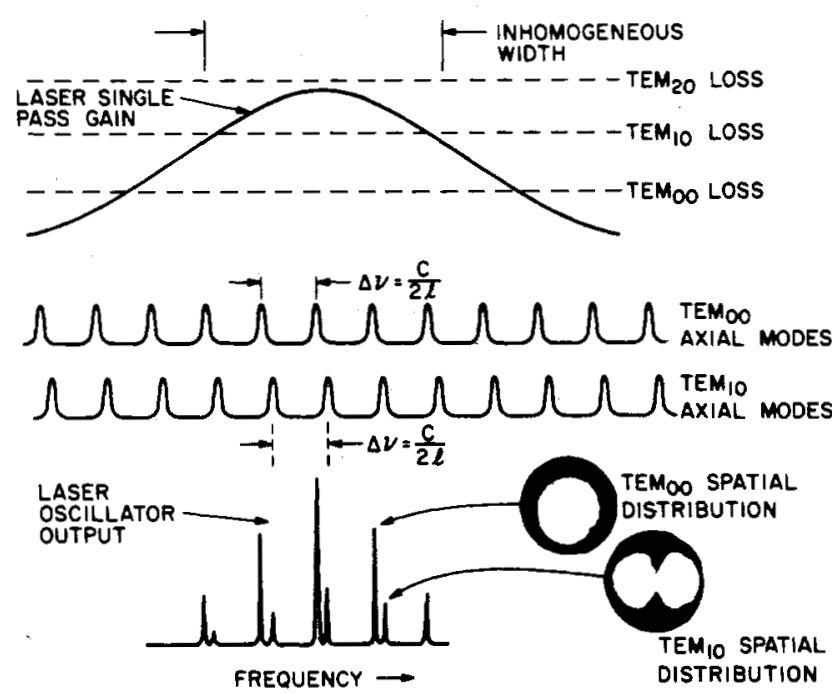

(b)

Fig. 2. Laser oscillator. (a) Regenerative optical oscillator. (b) Multimode operation of laser showing gain curve, cavity resonance, and cavity modes on which oscillation occurs.

losses for the two lowest order transverse modes. The spatial and temporal coherence of a laser source arises from the regenerative character of the combined laser gain medium and the optical cavity.

Review articles on lasers and on modes in optical resonators are to be found in [5]-[12]. Discussions of the laser oscillator and the statistical properties of its output are found in [13]-[17].

\section{B. Parametric Conversion Sources}

Parametric interactions were first considered by Lord Rayleigh [18], and they have been extensively utilized at microwaves [19]. There are many types of optical parametric interactions [20]-[23]; however, we shall discuss only the case involving three optical waves and the nonlinear polarizability of a noncentrosymmetric crystalline material. In such materials the second-order nonlinearity is of the form

$$
P_{i}=\sum_{j} \sum_{k} \chi_{i j k} E_{j} E_{k}
$$

where the $\chi$ are the second-rank tensor components of the nonlinear susceptibility, the $P$ are vector components of the generated optical polarization, and the $E$ are the vector components of the applied electric field. For any two applied optical fields at frequencies $v_{1}$ and $v_{2}$ fields will be generated in the material at both the difference frequency $\left|v_{1}-v_{2}\right|$ and the sum frequency $\left(v_{1}+v_{2}\right)$.

1) Difference Mixing (Parametric Amplification and Oscillation): Consider an intense optical wave $v_{p}$ (the pump) and a second wave of lower frequency $v_{s}$ (the signal) incident on a nonlinear material as depicted in Fig. 3(a). A wave $v_{I}$ (the idler) will be generated in the nonlinear medium at

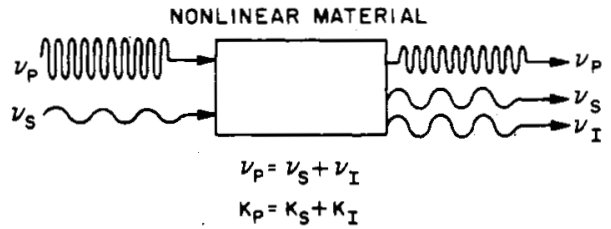

(a)

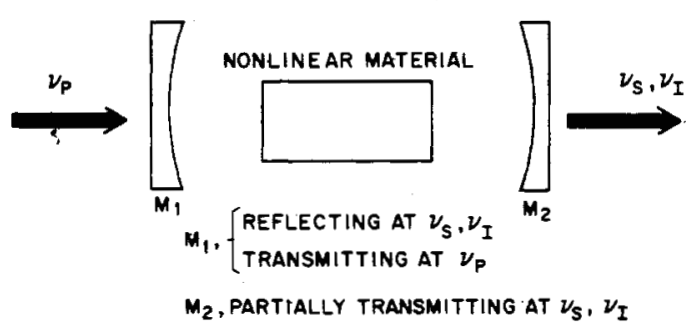

(b)

Fig. 3. Parametric down-conversion. (a) Schematic diagram of basic process, (b) Its use in doubly resonant (signal and idler frequencies resonated) optical parametric oscillator.

the difference frequency $v_{p}-v_{s}$. The three coupled waves satisfy the condition

$$
v_{p}=v_{s}+v_{I}
$$

which is the condition for conservation of energy. In order for the three waves to interact strongly, allowing an efficient power transfer between each other, momentum conservation or phase matching of the propagating wave vectors

$$
\boldsymbol{k}_{p}=\boldsymbol{k}_{\mathrm{s}}+\boldsymbol{k}_{I}
$$

is required. For the collinear case depicted in Fig. 3(a), (3) becomes

$$
n_{p} v_{p}=n_{s} v_{s}+n_{I} v_{I}
$$

where the $n$ are the refractive indices of the material at the respective frequencies. In Fig. 3(a) the signal wave beats with the pump wave through the nonlinear polarizability to produce the idler wave. As the idler travels through the nonlinear material it also beats with the pump wave to produce a polarization wave at the signal with just the proper phase to amplify the original signal wave. It follows that the signal and idler waves experience exponential gain at the expense of the pump wave. Since there is gain at the signal and idler frequencies, an oscillator can be built, as is shown in Fig. 3(b). Oscillation occurs when the pump power provided produces sufficient gain to overcome cavity losses at the signal and idler frequencies. With presently existing nonlinear materials, optical parametric oscillation is possible only by using a laser pump. For a given pump frequency and nonlinear material, oscillation occurs, in the collinear case, at those $v_{s}$ and $v_{I}$ which satisfy (2) and (4). Tuning of $v_{s}$ and $v_{I}$ can be achieved by utilizing means such as temperature or an electric field to change the refractive indices of the crystal. This type of oscillator does not use discrete energy levels and, since the nonlinear $\chi$ are approximately constant at optical frequencies, it is inherently broadly tunable. Optical parametric oscillators have recently been reviewed by Harris [24].

2) Sum Mixing (Second-Harmonic Generation): In sum frequency generation, two intense optical waves of frequencies $v_{1}$ and $v_{2}$ interact in the nonlinear medium to 
produce a third wave of frequency $v_{3}=v_{2}+v_{1}$. As in our previous discussion strong interaction occurs only if there is momentum conservation, $\boldsymbol{k}_{3}=\boldsymbol{k}_{1}+\boldsymbol{k}_{2}$. In contrast to difference mixing, there is no possibility for achieving electronic gain and only power conversion from waves $v_{1}$ and $v_{2}$ to $v_{3}$ will occur. Sum mixing of lasers in a nonlinear material is another way of generating new source frequencies. The most common example is second-harmonic generation for which $v_{1}=v_{2}$ and $v_{3}=2 v_{1}$. A detailed discussion of nonlinear optical sum frequency generation can be found in [25], [26] and its practical usage with $\mathrm{CW}$ lasers in [27]-[29].

\section{Control of the Spatial and Temporal CHARACTERISTICS OF OPTICAL OSCILLATORS}

The spatial and temporal characteristics of an optical oscillator are important in communication systems. For efficient optical heterodyne detection, the source and local oscillator should have the same transverse mode distribution, preferably the lowest order $\mathrm{TEM}_{00}$ mode. Singlefrequency operation (i.e., operation on a single axial mode) is required in some cases. In a pulse-code modulation (PCM) system [30], an oscillator which emits repetitive pulses is most useful. In this section some representative techniques which have been developed to control the spatial and temporal behavior of optical oscillators are discussed.

\section{A. Transverse Mode Selection}

In the laser oscillator in Fig. 2, transverse mode selection can be achieved by selectively increasing the loss of one transverse mode relative to another by introducing an appropriate obstacle into the cavity. For example, the TEM ${ }_{00}$ can be suppressed if a small absorbing wire is placed at one of the mirrors in a direction coincident with the field null of the $\mathrm{TEM}_{10}$ mode. More typically, operation in the $\mathrm{TEM}_{00}$ mode and suppression of all other modes including the TEM $_{10}$ mode is desired. Calculations show that the lowest order modes always have lower diffraction losses than higher order modes. As a result, if the diameter of the resonator is sufficiently constricted at some point with an aperture stop, only the lowest order transverse mode can oscillate, all others being prohibited by excessive diffraction loss. Other methods of achieving single transverse mode operation of optical oscillators are mentioned by Kogelnik [31]. The inclusion of the effect of transverse modes on the saturation of the gain medium has been theoretically treated by Bloom [32] and Fox and $\mathrm{Li}$ [33].

\section{B. Axial Mode Selection and Stabilization}

Operation on a single axial mode can be realized in a number of different ways utilizing a variety of multiplemirror optical resonator configurations to achieve the appropriate limitations of resonances [34]. One representative method which has been employed utilizes an intracavity Fabry-Perot etalon, as is depicted in Fig. 4. The etalon is inserted at the position of the beam waist in the cavity with a tilt angle that provides sufficient reflection loss to prevent oscillation of all the unwanted axial modes of the long cavity. Oscillation occurs at a transmission maxi-

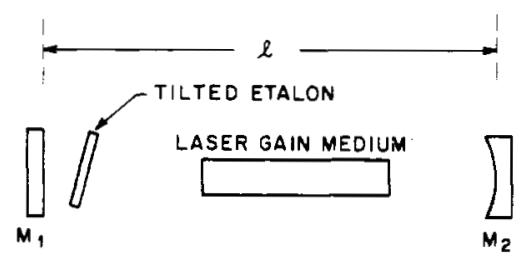

(a)

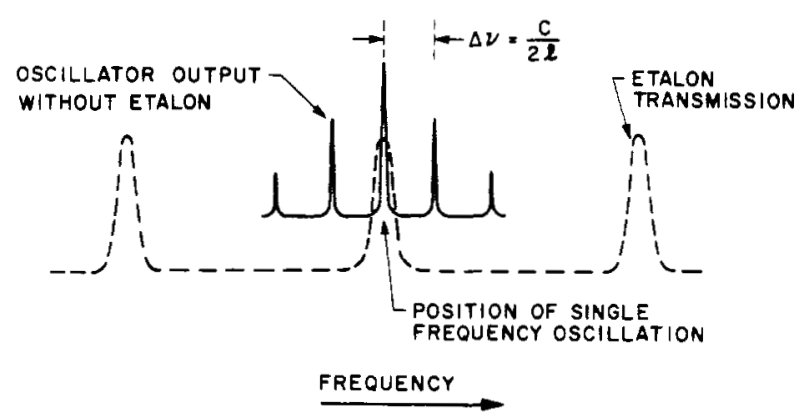

(b)

Fig. 4. Single frequency operation laser using tilted intracavity etalon. (a) Schematic diagram. (b) Effect of etalon transmission on selection of single axial resonance.

mum of the etalon. This maximum is made to coincide with a laser cavity mode near the center of the gain profile. The optimum design of the tilted etalon mode selector and its use for stabilizing the output of an optical oscillator has been described by Danielmeyer [35]. The frequency stabilization of gas lasers has been reviewed by Polanyi and Tobias [36].

\section{Cavity Modulation Techniques}

A variety of methods utilizing intracavity modulation techniques have been developed which permit continuously pumped optical oscillators to be repetitively pulsed. Three such techniques, which have been historically referred to as mode locking, $Q$ switching, and cavity dumping, will be discussed.

In addition, intracavity modulation has been employed by Harris [37] to produce FM or single-frequency outputs. Since a discussion of this work will not be given in this paper, the reader is directed to [37].

1) Mode Locking: A single transverse, multiaxial mode laser can be repetitively pulsed by introducing an intracavity loss or phase modulator which is driven at a frequency equal to the frequency separation, $\Delta v=c / 2 l$, between axial modes. The effect of the perturbation is to couple the axial modes in an AM manner. For a large number $N$ of locked modes the oscillator has an oscillation envelope which is a pulse train with period $T=2 l / c$, pulsewidth $\tau=T / N$, and a peak power $N$ times the average power. For an oscillator with $l=30 \mathrm{~cm}$ and $N=20, T=2 \times 10^{-9}$ second and $\tau=10^{-10}$ second. Mode-locked pulsewidths of $500 \mathrm{ps,} 200 \mathrm{ps}$, and $50 \mathrm{ps}$ have been obtained, respectively, from $\mathrm{He}-\mathrm{Ne}$ [38], Ar [39], and Nd: YAlG [40] lasers. Such mode-locked sources are useful in high-data-rate optical PCM systems [30]. The experimental and theoretical aspects of modelocked lasers have been studied extensively and are discussed in [37]-[46].

2) $Q$ Switching and Cavity Dumping: Although mode locking can be extended to low. repetition rates by using 


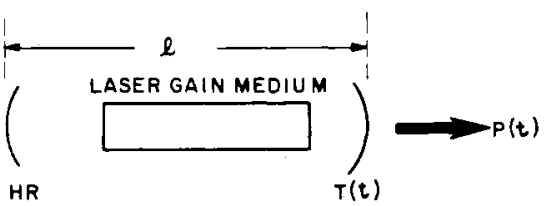

(a)

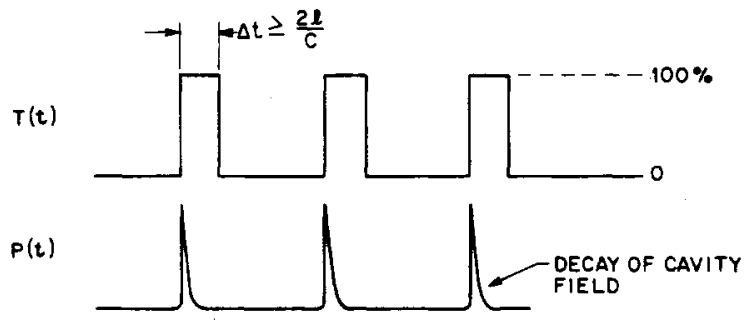

(b)

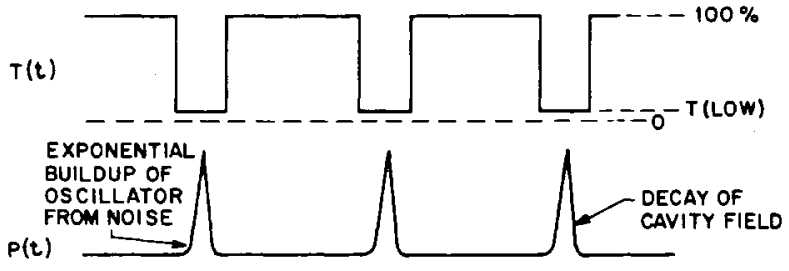

(c)

Fig. 5. Repetitive cavity dumping and $Q$ switching of laser oscillator. (a) Laser oscillator which has time-dependent mirror transmission; in practice, this is effectively accomplished using an intracavity modulator and a mirror whose transmission is time-independent. (b) Cavitydumping operation cycle. (c) $Q$-switched operation cycle.

long optical cavities, it is somewhat impractical at repetition rates below $30 \mathrm{MHz}$. Up to $100 \mathrm{kHz}, Q$ switching is effective with a laser such as $\mathrm{Nd}$ : YAlG; from $100 \mathrm{kHz}$ to $30 \mathrm{MHz}$ cavity dumping is preferred. The latter two techniques can be described with the aid of Fig. 5 where the transmission of the output mirror of the oscillator is assumed to be time dependent in response to a drive signal. In practice, this can be effectively accomplished using an intracavity modulator and a mirror whose transmission is time independent.

In cavity-dumped operation [Fig. 5(b)] the oscillator stores energy in the optical cavity during the interpulse period when the output mirror transmission is zero. For the case in which maximum peak output power is desired, the transmission is switched to 100 percent (for a time interval $\geq 2 l / c$ ) at a time when the optical cavity has maximum stored energy. As a result, all the stored energy of the cavity is dumped and the output is a narrow pulse whose peak power is equal to the maximum internal circulating power. Restoration of the mirror transmission to zero permits a repetition of the cycle. In general, to maximize the average output power for a given repetition rate the mirror transmission in the high transmission state is less than 100 percent and is held for times longer than $2 l / c$. The technique of cavity dumping was first proposed by Vuylsteke [47] for pulse pumped lasers and applied to $\mathrm{CW}$ gas lasers by Steier [48]. A theoretical discussion of repetitive cavity dumping and, in particular, its application to the Nd: YAlG laser has recently been given by Chesler and Maydan [49].

The operation cycle for $Q$ switching is shown in Fig. 5 (c). Here the mirror transmission is 100 percent during most of the cycle, so as to prevent oscillation and to allow the energy to be stored in the laser medium (in contrast to the intercavity flux storage used in the cavity-dumping technique). During this period, the single-pass laser gain builds up to a value which exceeds the mirror transmission in the low transmitting state. The mirror is switched to its low transmission state for a period sufficient to allow the oscillator to build up from noise and discharge the inversion energy stored in the gain medium. This period depends on the round-trip transit time of light in the cavity and the maximum single-pass unsaturated gain achieved. $Q$ switching was first proposed by Hellwarth [50] and applied to pulsed solid-state lasers by McClung and Hellwarth [51]: Theoretical and experimental investigations of repetitively $Q$ switched, continuously pumped lasers are reported in [52]-[55].

\section{GAS LASERS}

Gas lasers are, perhaps, more difficult to discuss as a group than either semiconductor or solid-state lasers because they possess more diverse properties. Their wavelengths span the range from the shortest (Ne IV, $0.2358 \mu$ [56]) to the longest (ICN) $774 \mu$ [57]) among lasers. Their $\mathrm{CW}$ or average power outputs also range from the highest ( $>9 \mathrm{~kW}$ from $\mathrm{CO}_{2}$ [58]) to the "lowest" (that is, the lowest of practical interest, the stabilized $\mathrm{He}-\mathrm{Ne}$ laser used for metrological applications [59]). They utilize an amazing variety of pumping methods, e.g., electrical excitation in the form of glow discharges, both RF and dc excited ( $\mathrm{He}-\mathrm{Ne}$ and $\mathrm{CO}_{2}$ ); arc discharges, $\mathrm{RF}$, dc, and pulsed (ion lasers); special discharges (the ultraviolet $\mathrm{N}_{2}$ laser [60] and recent types of $\mathrm{CO}_{2}$ lasers [61]); optical excitation ( $\mathrm{He}$ lamp pumping of $\mathrm{Ce}$ vapor [62] and $\mathrm{CO}$ flame pumping of $\mathrm{CO}_{2}$ [63]); chemical excitation (the HF laser [64] and the DF pumped $\mathrm{CO}_{2}$ laser [65]); thermal excitation (expansioninverted $\mathrm{CO}_{2}$ [66]); and various combinations (photodissociated $\mathrm{CH}_{3} \mathrm{I}$ [67], electrically formed $\mathrm{HBr}$ [68], etc.). Many of these gas lasers could be used for communication, but because of the wide selection of operational characteristics available to the user, only the most useful few have received the serious attention and development necessary to realize performances approaching their optimum as practical optical communication sources. Even the few selected for development exhibit too wide a variety of characteristics to receive a complete discussion here. We will have to be content with examples of the most familiar types used for communication and restrict ourselves to considering the characteristics most important for communication, i.e., power output, life, efficiency, mode control, frequency control, and growth potential.

The three examples chosen are the three most popular gas lasers now in use, i.e., the $\mathrm{He}-\mathrm{Ne}$ laser, the $\mathrm{Ar}$ ion laser, and the $\mathrm{CO}_{2}$ laser. Table $\mathrm{I}$ lists the important characteristics of these lasers: the "age," the principal wavelength(s), the nature of the active medium (for the most common excitation methods; the $(E)$ or $(H)$ after radio frequency (RF) indicates the principal RF coupling method used), and the usual range of average or $\mathrm{CW}$ output powers and efficiencies. A more detailed description of each laser 
TABLE I

Characteristics of Gas LASERS

\begin{tabular}{|c|c|c|c|}
\hline & $\mathrm{He}-\mathrm{Ne}$ & Ar II & $\mathrm{CO}_{2}$ \\
\hline Years since "Discovery" & 8.5 & 6.5 & 5.5 \\
\hline \multirow[t]{2}{*}{ Wavelength $(\mu)$} & 0.633 & 0.488 & 10.6 \\
\hline & $\begin{array}{l}1.15 \\
3.39\end{array}$ & $\begin{array}{c}0.515 \\
(0.45-0.53)\end{array}$ & $\begin{array}{c}9.6 \\
(9.6-10.8)\end{array}$ \\
\hline $\begin{array}{l}\text { Medium } \\
\text { Current density }\left(\mathrm{A} / \mathrm{cm}^{2}\right) \\
\text { Excitation }\end{array}$ & $\begin{array}{c}\text { Glow discharge in } \mathrm{He}-\mathrm{Ne} \text { mix } \\
0.05-0.5 \\
\text { dc, RF }(E)\end{array}$ & $\begin{array}{c}\text { Arc discharge in pure Ar } \\
100-2000 \\
\text { dc, } \operatorname{RF}(E, H)\end{array}$ & $\begin{array}{c}\text { Glow discharge in } \mathrm{CO}_{2}-\mathrm{N}_{2}-\mathrm{He} \\
0.01-0.1 \\
\text { dc, RF }(E)\end{array}$ \\
\hline Power (at wavelength) & $(0.633)$ & (multicolor) & $(10.6)$ \\
\hline Best laboratory (watts) & 1 & 100 & 9000 \\
\hline Best commercial (mW) & 100 & 20 & 1000 \\
\hline Lowest commercial $(\mu \mathrm{W})$ & 100 & 1 & 1 \\
\hline Efficiency (percent) & $0.001-0.1$ & $0.01-0.2$ & $1-20$ \\
\hline
\end{tabular}

type including the technical problems most relevant to their use as optical communications sources is given in the following subsections.

\section{A. He-Ne Lasers}

It is reasonable to say that the $\mathrm{He}-\mathrm{Ne}$ laser has reached its peak performance because of its age and the relatively "nice" nature of its active medium, a noble gas glow discharge. The principal wavelengths of interest for communication are the red line at $0.6333 \mu$ and the $3.39-\mu$ infrared line; some applications have also been found for the $1.15-\mu$ line. The commercially available output power of $100 \mathrm{~mW}$ for the red line listed in Table I is not likely to increase for purely practical reasons. Almost 1 watt has been obtained in a laboratory type He-Ne tube [69]; however, the tube was over 5 meters long, while a 1 watt Ar ion laser is typically less than 1 meter long. The "lowest commercial power" listed for the He-Ne laser is $\sim 100 \mu \mathrm{W}$ obtainable from small single-frequency stabilized units [59]. The efficiency figures shown correspond to the range of output power and are also not likely to improve.

The power output and efficiency figures for the 3.39- $\mu$ line are roughly 25 percent of those given for the $0.633-\mu$ line in any given tube size. This is simply the ratio of photon energies for the two lines and reflects the fact that they share a common upper level. The gain coefficient for the $3.39-\mu$ line is much higher than for the $0.633-\mu$ line (all other things being equal, the gain coefficient being proportional to $\lambda^{3}$ ). As a consequence, the 3.39- $\mu$ laser discharge tube can be used practically as a single-pass amplifier while the $0.633-\mu$ laser is confined to oscillator service by its very low gain coefficient, typically a few percent per meter.

$\mathrm{He}-\mathrm{Ne}$ lasers exhibit a sharp optimum value in power output as a function of discharge current; a further increase in input power does not yield a corresponding increase in output power. This optimum operating point occurs for quite fundamental reasons, i.e., the neon upper laser level is populated by resonant collisions with excited helium. atoms

$$
\mathrm{He}\left(2 s^{1} S_{0}\right)+\mathrm{Ne} \rightarrow \mathrm{He}+\mathrm{Ne}\left(5 s^{\prime}\left[\frac{1}{2}\right]_{1}^{0}\right)
$$

for the 0.633- and 3.39- $\mu$ lines. Because the excited helium state involved is metastable (i.e., it cannot decay by the spontaneous emission of a photon), it is both created and destroyed by electron or wall collisions. Thus the helium metastable population at first increases with discharge current (roughly proportional to plasma electron density), but soon saturates when the creation and destruction rates by electron collision are equal. The neon upper laser level follows the helium metastable population closely through process (5) and thus also saturates with current. On the other hand, the lower neon laser levels $\mathrm{Ne}\left(3 p^{\prime}[3 / 2]_{2}\right)$ for the $0.633-\mu$ line and $\mathrm{Ne}\left(4 p^{\prime}[3 / 2]_{2}\right)$ for the $3.39-\mu$ line, have allowed transitions to levels below them and are thus primarily destroyed by spontaneous emission (independent of electron density). However, these lower levels are populated by electron collision. For this reason their populations continue to increase with increasing current. Since the laser gain is proportional to the population difference, a maximum gain will occur at a somewhat lower current than that necessary to saturate the helium metastable population [70]. This optimum discharge current density is relatively modest $\left(0.05-0.5 \mathrm{~A} / \mathrm{cm}^{2}\right)$ in typical He-Ne lasers, so that the associated technology has, in large measure, also "saturated" with simple glass envelope tubes and hot or cold cathodes with small current loading. The modest technology requirements imposed by the neon-sign-like active medium allowed a fairly complete mapping of the optimum operating condition very early in the game. Subsequent engineering efforts have been devoted primarily to extending tube life by reducing gas cleanup (largely through the proper choice of cathode type) and improving the optical elements within the cavity (mirrors, windows). Electrodeless RF discharges were used early in the research stage, but they have almost disappeared because of their relatively severe gas cleanup rates. Fig. 6 shows a typical small $\mathrm{He}-\mathrm{Ne}$ laser discharge tube. The glass discharge capillary extends completely through the hollow cold cathode. The anode (weld ring) is evident at the left end of the tube and a getter is suspended from the cathode pins. Brewster's angle optical windows are sealed on each end 


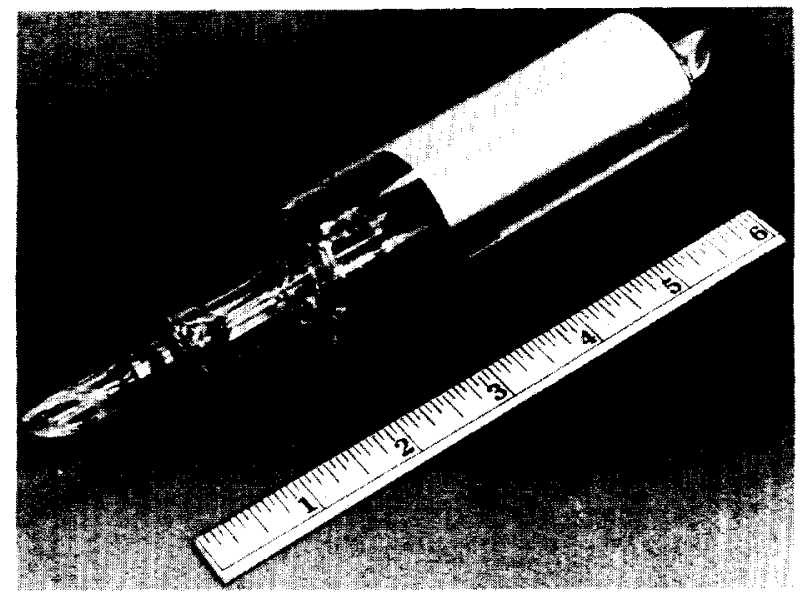

Fig. 6. Typical small $(0.5 \mathrm{~mW})$ external-mirror He-Ne laser.

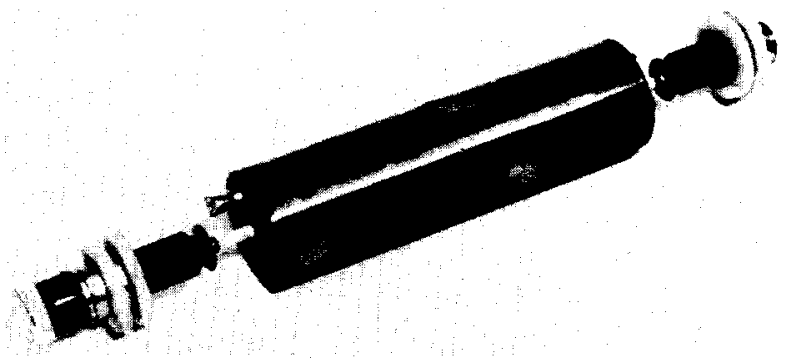

(a)

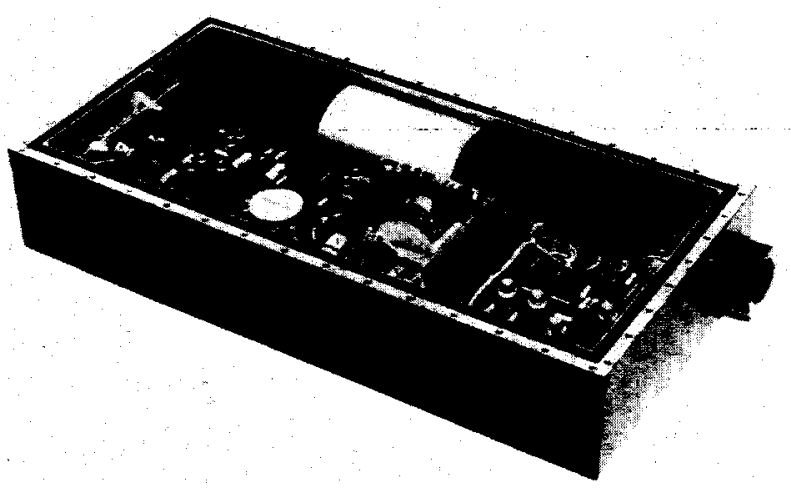

(b)

Fig. 7. (a) Metal-ceramic internal-mirror He-Ne lasers for space-qualfied systems. (b) Integrated space-qualified He-Ne laser package ( 5 $\mathrm{mW}$ ) with all solid-state power conditioning system. Prime power requirements are 30 watts at $24-32$ volt dc.

with low vapor pressure epoxy. While this sealing method may seem undesirable to those experienced in the vacuumtube art, it remains the best compromise to date when window cleanliness and optical quality, vacuum properties and fabrication cost are balanced. Tubes using these construction techniques have given operation on life tests in excess of 22000 hours and show a statistical mean time between failures (MTBF) in excess of 28000 hours [71].

A somewhat more challenging engineering development is shown in Fig. 7, a metal-ceramic He-Ne tube designed for use in space [72]. The discharge tube shown in Fig. 7(a) has mirrors internal to the vacuum envelope and thus requires no intracavity sealed windows, although an internal Brewster's angle window (no vacuum seal) is used in the version shown in order to provide a plane-polarized output.
The flexible bellows on each end allow mirror motion and also serve as anodes. Only small portions of the ceramic capillaries are visible at each end of the tube, but they extend into the center of the large hollow cold cathode. Because no epoxy is used in this tube, a high temperature bake-out schedule can be used to ensure complete outgassing of all elements and assure long operating life. The space-packaged laser is shown in Fig. 7(b). The discharge tube is contained within the beryllium cylinder, located at the top of the package; this cylinder maintains the optical alignment of the laser mirrors. The remainder of the packages contains the solid-state power conditioning and telemetry electronics. The specification on this tube is $5-\mathrm{mW}$ output power at $0.633 \mu$ for a minimum of 10000 hours in a spacecraft environment.

Because of its high state of development, special purpose $\mathrm{He}-\mathrm{Ne}$ lasers such as the space-qualified unit previously described, are now straightforward engineering projects. The various techniques for controlling the spatial and temporal characteristics referred to in Section III, with the exception of $Q$ switching, have all been used successfully on $\mathrm{He}-\mathrm{Ne}$ lasers; in fact, these techniques were all originally demonstrated with $\mathrm{He}-\mathrm{Ne}$ lasers. The remaining challenges for He-Ne laser development is primarily improvement in manufacturing economy. Fully automated production should be able to reduce the price to the level of a conventional glass electron tube, provided large volume applications develop. The availability of such cheap lasers should open the way for new communications uses.

\section{B. Ar-Ion Lasers}

If $\mathrm{He}-\mathrm{Ne}$ lasers are the "glass receiving tubes" of the laser world, CW ion lasers are the klystrons and TWTs in terms of complexity. Despite intensive research and development efforts, the peak in ion laser performance has not yet been reached for several reasons. First, the laser medium is much harder to handle than the $\mathrm{He}-\mathrm{Ne}$ glow discharge. The ion laser medium is typically a low-pressure (less than 1 torr), small capillary arc (1 to $10 \mathrm{~mm}$ in diameter), with current densities ranging from 100 to more than $10000 \mathrm{~A} / \mathrm{cm}^{2}$ (the latter figure refers to pulsed operation). This is two to four orders of magnitude larger than the densities required by the He-Ne laser. Second, the output power continues to increase with increasing current density; at present this value is limited by discharge technology (2000 A/ $\mathrm{cm}^{2}$ for $\mathrm{CW}$ operation). Thus the technology has not yet "saturated;" the solution of a problem which allows an increase in performance merely leads to new technological problems at the new power level.

This property of the ion laser can be understood from first principles in much the same way as the $\mathrm{He}-\mathrm{Ne}$ laser [73]. In the ion laser, the upper laser levels (the $4 p$ ionic states) are populated by a combination of direct electron collision and radiative decay from higher lying levels populated by electron collision. Although the details are not known completely quantitatively, it is certain that at least two successive collisions are required, and that the last collisional step involves the impact of an electron and an ion. 
As a consequence, the upper level population varies as the product of electron density and ion density, or approximately as electron density squared, since the plasma has charge neutrality. Over the range of plasma parameters typical of the ion laser the electron density is approximately proportional to the discharge current density, so that the upper level population should vary as the square of the discharge current density. This has been confirmed experimentally by spontaneous emission measurements. The lower laser levels (the $4 s$ ionic states) are populated in much the same way, and thus the population difference varies as the square of the current density.

There are processes which can upset this simple picture, such as depletion of the neutral gas from the discharge region of the tube by plasma processes of heating, or trapping of the spontaneous ultraviolet emission which depopulates the lower laser level. However, thermal depletion of the neutral gas in the discharge active region is only now being observed at the highest current densities $(>1000$ $\mathrm{A} / \mathrm{cm}^{2}$ ), and radiation trapping is apparently only a problem in pulsed ion lasers, where ion heating does not have time to occur. Thus for $\mathrm{CW}$ ion lasers designed for the 0.1 to 100 watt output range, the output continues to increase with increasing current limited only by the tube construction techniques. An empirically derived relation for the power output per unit volume of active discharge is [73]

$$
P / V=10^{-5} J^{2}
$$

when $P$ is in watts, $V$ in $\mathrm{cm}^{3}$, and $J$ in $\mathrm{A} / \mathrm{cm}^{2}$. In this expression, $P$ is the multimode multicolor output of the laser. If single-color operation at $0.488 \mu$ or $0.515 \mu$ is selected by using a dispersive intracavity element, $30-40$ percent of this power can be obtained. If TEM $_{00}$ operation is required, some sacrifice in power is usually experienced because of the additional diffraction losses that are usually introduced to select $\mathrm{TEM}_{00}$ operation; however, the degradation depends on the size of the laser and the cleverness of the technique used. Usually, 70-80 percent of the multimode power can be obtained in $\mathrm{TEM}_{00}$ operation. Selection of a single longitudinal mode can also be accomplished by any one of the several techniques referred to in Section III-B. Because of a strong tendency to behave as a homogeneously broadened line, especially at high power levels, almost all of the multimode power can be obtained in a single longitudinal mode except that part lost due to additional intracavity elements.

Ion laser technology has progressed through several stages with corresponding increases in power output and tube life. Early discharge tubes were made of fused silica, similar to the present $\mathrm{He}-\mathrm{Ne}$ tubes, except that water cooling was required. Actually, the very first $\mathrm{CW}$ ion laser [74] was not water cooled but was operated with incandescent quartz walls. The need for cooling was painfully evident after a few minutes of operation. Fig. 8 shows the cathode throat region of a typical quartz discharge tube after several hours of operation. Localized sputtering and decomposition of the capillary wall has occurred, especially at the entrance

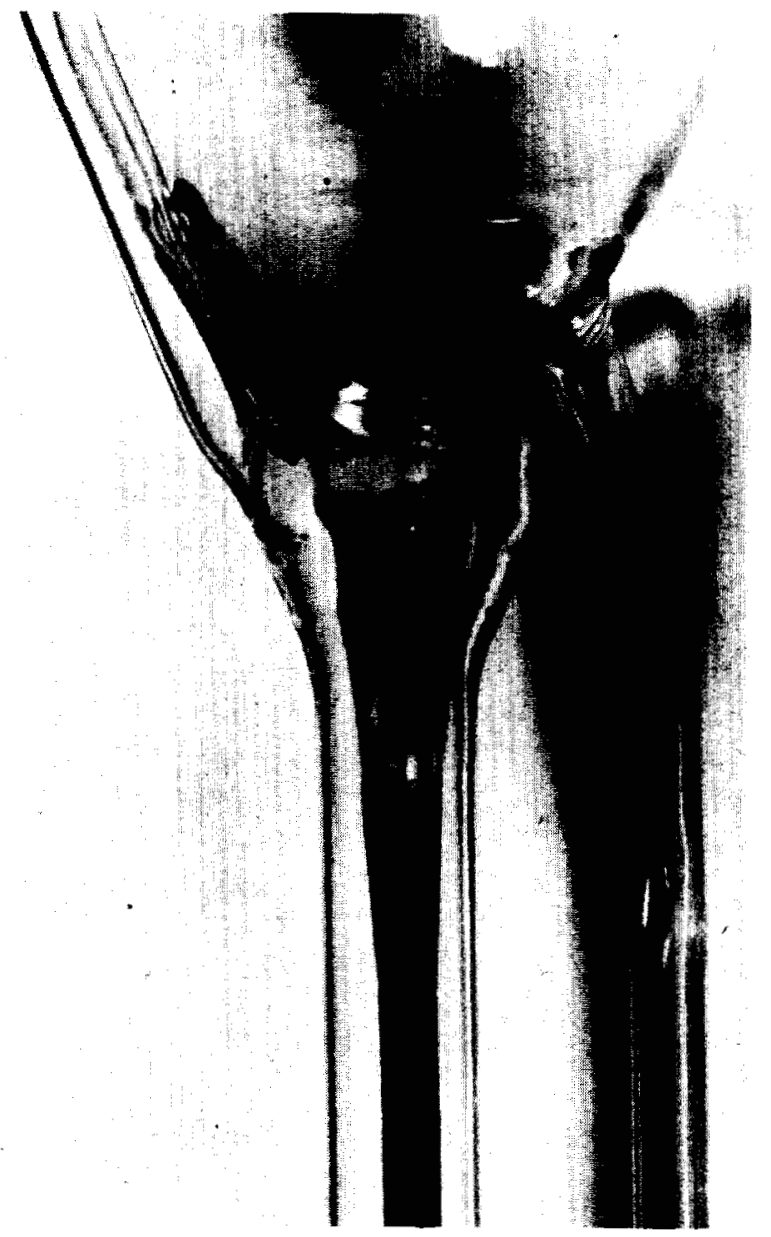

Fig. 8. Cathode throat region of typical $\mathrm{CW}$ ion laser silica discharge tube after 100 hours of operation. Discoloration is silicon or silicon monoxide from ion-decomposed $\mathrm{SiO}_{2}$.

of the uniform bore region. After a few hundred hours of operation, the same sputtering damage will be seen along the entire bore, and the entrance region may be eroded completely through the capillary wall (typically $1-\mathrm{mm}$ thick). The sputtering process not only limits tube life by physical damage, but also cleans up the gas in the discharge by burying it in the walls in exactly the same manner as the noble gases are pumped by an ion vacuum pump. Typical design limits for $\mathrm{CW}$ quartz bore ion laser tubes are 100 to 150 watts of input power per centimeter of length, 200 to 500 hours to ultimate end of life, and $10 \mu \cdot 1 / \mathrm{h}$ gas cleanup rate. Within these limits, compact lasers can be built with a few watts output, provided a gas replenishing system or a very large gas reservoir is supplied.

Several different methods have been tried in efforts to improve the characteristics of quartz bore tubes. Among the different dielectric bore materials that have been tried are boron nitride, alumina, and beryllia. Of these, beryllia (BeO) has proven to be the most successful. Standard metal-ceramic brazing techniques have been used to make beryllia bore discharge tubes which exhibit very low sputtering and gas cleanup rates; a dissipation capability of over $300 \mathrm{~W} / \mathrm{cm}$ has been demonstrated to date. Fig. 9 shows a small $\mathrm{BeO}$ bore (25-cm active length) discharge tube which produces over an 8-watt output. This tube 


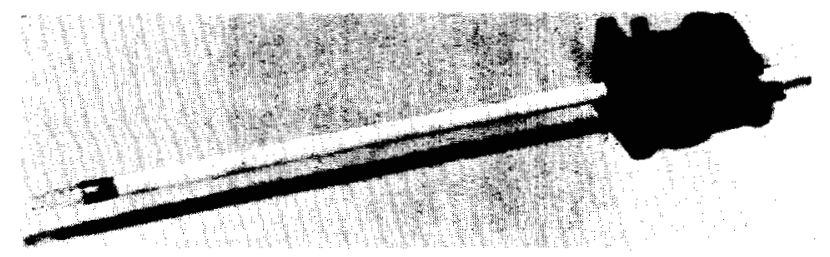

Fig. 9. Typical metal-ceramic BeO bore Ar ion laser discharge tube. 11 -inch active region of this tube produces over 5 watts of output.

utilizes the all metal-ceramic construction techniques and impregnated tungsten cathode so familiar in modern microwave tubes. A gas return path, to equalize the gas pressure differential between cathode and anode generated by discharge processes, is incorporated as an integral part of the $\mathrm{BeO}$ structure. A recent life test in a similar $\mathrm{BeO}$ tube ran over 800 hours $^{1}$ (cycled in 6- to 9-hour operating intervals) with no measurable change in gas pressure. The estimated gas cleanup rate was less than $0.01 \mu \cdot 1 / \mathrm{h}$ [75].

It is also possible to make the bore walls out of electrically conducting materials. If the material is distributed along the bore in short segments, each insulated from the next, the discharge is substantially unaffected by the local short circuit [76]. Low-sputtering refractory materials such as tungsten [73], molybdenum [76], tantalum, and graphite [77] have been used in radiation-cooled arrangements; copper [78] and aluminum [79] have also been used in water-cooled assemblies. Tungsten has the lowest sputtering yield of the refractory metals, and has also been used as a bore material in high-performance ion lasers. Disk bore structures have been used at input power levels of $500 \mathrm{~W} / \mathrm{cm}[80]$.

Fig. 10 shows a number of tungsten disks assembled in a stacked structure which is then slipped inside the vacuum envelope. The discharge and laser characteristics of this structure of relatively thin conducting disks separated by relatively large gaps are also substantially the same as those of a continuous dielectric wall. The high emissivity of the material plus the stacked-disk structure allows very high power-per-unit-length operation with radiation cooling to free space or a surrounding water jacket. Graphite can also be used in this fashion, but it has the disadvantage of low mechanical strength with a resultant tendency to form powder, a very undesirable property for devices containing optical surfaces. The low sputtering yield of carbon and its low cost make it an attractive material for laboratory-type tubes in which carbon dust can lie undisturbed in the bottom of the envelope, but it is not suitable for tubes which must be moved, operated in a vertical position, or pass a shake test.

Clearly, there will be continued developments in ion laser technology leading to higher output power, more compact packaging, and less expensive units. New wavelengths, particularly in the ultraviolet [80], will become increasingly more practical, although they will undoubtedly be used more in scientific instrumentation rather than communica-

\footnotetext{
${ }^{1}$ The life test was terminated by a need for the test equipment and space (for a new tube), not by any change in operating characteristics.
}

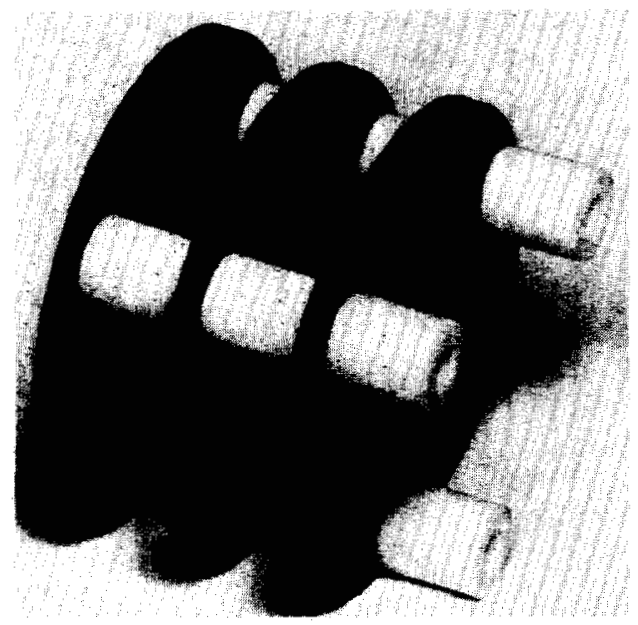

Fig. 10. Stacked tungsten disks used to form radiation-cooled bore of a high-power ultraviolet ion laser.

tion. It is not likely that ion laser efficiency will increase significantly over the 0.1 to 0.2 percent maximum demonstrated to date.

\section{C. $\mathrm{CO}_{2}$ Lasers}

The $\mathrm{CO}_{2}$ laser is the youngest of the three types listed in Table I. Although laser oscillation was first obtained [81], [82] on wavelengths in the $00^{\circ} 1 \rightarrow 10^{\circ} 0$ band of $\mathrm{CO}_{2}$ at about the same time as the Ar ion laser was demonstrated, it was approximately a year before the high-power highefficiency aspects were fully realized [83] and serious device development began. The nature of the active medium of a $\mathrm{CO}_{2}$ laser is similar in some respects to that of the $\mathrm{He}-\mathrm{Ne}$ laser. A glow discharge is used with about the same input power per unit length. The electric fields in the plasma tends to be somewhat higher for a given diameter discharge (by a factor of 3 or so) because of the higher operating pressure and the electronegative character of the gas mixture. Several methods of excitation have been used successfully, including hot- and cold-cathode de discharges, RF discharges, and microwave discharges; however, the most common method now in use is the same as that used in the $\mathrm{He}-\mathrm{Ne}$ laser, i.e., the cold-cathode dc discharges. For 1- to 100 -watt output $\mathrm{CO}_{2}$ lasers, glass or quartz envelope tubes very much like those used in the $\mathrm{He}-\mathrm{Ne}$ laser are usually employed, with the main differences being the addition of a water cooling jacket; $\mathrm{NaCl}, \mathrm{KCl}, \mathrm{GaAs}$, or $\mathrm{Ge}$ windows instead of fused silica; and a somewhat larger diameter (1 to $2 \mathrm{~cm}$ instead of 2 to $4 \mathrm{~mm}$ ).

Despite the apparent similarity of construction, the technology problems are quite different for the $\mathrm{CO}_{2}$ and $\mathrm{He}-\mathrm{Ne}$ lasers. Higher power $\mathrm{CO}_{2}$ lasers ( $>100$ watts) which employ continuous gas flow are commonly made of bolttogether glass pipe of the type used for food or chemical plumbing. A laser oscillator with a $\mathrm{CW}$ output in excess of $9 \mathrm{~kW}$ has been made using this construction technique [58]; laser power amplifiers with average power outputs $>1 \mathrm{~kW}$ when driven by single frequency, TEM ${ }_{00}$ oscillators have also been made in this way by Smith and Forster [84], as shown in Fig. 11. 


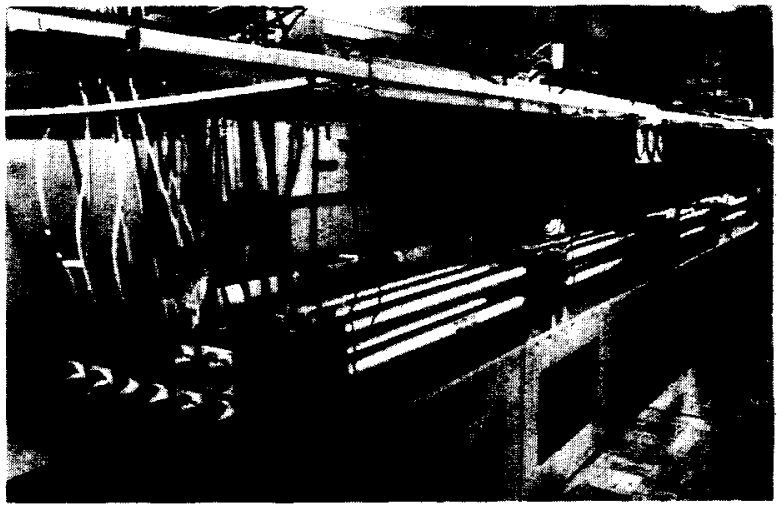

Fig. 11. Early breadboard version of $1.5-\mathrm{kW}$ average power. $15-\mathrm{kW}$ peak power MOPA $\mathrm{CO}_{2}$ laser system.

Specific output powers $>1 \mathrm{~W} / \mathrm{cm}^{3}$ have been obtained in small tubes, although larger diameter tubes usually produce no more than $0.1 \mathrm{~W} / \mathrm{cm}^{3}$. A typical 10-watt output laser might have a discharge tube $1 \mathrm{~cm}$ in diameter by 50 $\mathrm{cm}$ in length and require 100 watts input power $(5 \mathrm{kV}$ at $20 \mathrm{~mA}$ ). The high efficiency is primarily a consequence of the fact that both upper and lower laser levels lie very close to the ground state. Because of this low-lying position, almost all of the higher energy levels excited by the discharge funnel down to the upper laser level by cascade, and very little energy is wasted in relaxing the lower level to the ground state. Because the $\mathrm{CO}_{2}$ laser exhibits relatively higher gains in larger diameter tubes than either $\mathrm{He}-\mathrm{Ne}$ or Ar II lasers, it is relatively simple to obtain higher power by increasing the tube volume. The $1-\mathrm{kW}$ value listed in Table I was obtained in about 20 meters of discharge and the $9 \mathrm{~kW}$ value in about 200 meters (folded into a more reasonable length, of course).

More recently, a basically new type of $\mathrm{CO}_{2}$ laser configuration was reported, one which uses a fast-flowing gas mixture to obtain high output power per unit volume [85]. An output in excess of $1 \mathrm{~kW}$ was reported for a 1-meter long $\mathrm{CO}_{2}$ oscillator. The specific output from this device is substantially $>1 \mathrm{~W} / \mathrm{cm}^{3}$; however, a better figure of merit for this class of fast-flow lasers is the power output per volumetric flow rate. Outputs of $1 \mathrm{~W} / \mathrm{ft}^{3} / \mathrm{min}$ have already been demonstrated. This number is in good agreement with the theory for fast-flow systems.

In all $\mathrm{CO}_{2}$ lasers, mixtures of $\mathrm{CO}_{2}$ with several other gases are employed. It is possible to make a "pure" $\mathrm{CO}_{2}$ laser, but in reality the "pure" $\mathrm{CO}_{2}$ is quickly dissociated by the discharge into an equilibrium mixture of $\mathrm{CO}_{2}, \mathrm{CO}$, and $\mathrm{O}_{2}$ (or $\mathrm{O}, \mathrm{O}-$ ). The $\mathrm{CO}$ then populates the upper laser level by resonant collision in the same manner that helium atoms pump the $\mathrm{Ne}$ upper laser level in the He-Ne laser. More efficient pumping is possible if nitrogen is added to the discharge, since the net transfer of excitation from the $\mathrm{N}_{2}$ vibrational states to the $\mathrm{CO}_{2}$ upper laser level is better than from the $\mathrm{CO}$ vibrational states. The $\mathrm{N}_{2}$ or $\mathrm{CO}$ molecules present are excited by collisions with the discharge electrons; at high current densities the excited $\mathrm{N}_{2}$ and $\mathrm{CO}$ also can be destroyed by electron collision, with the resultant formation of a current-independent equilibrium pop- ulation. However, this does not occur in the usual operating range of sealed-off or slow-flow $\mathrm{CO}_{2}$ lasers. Within this range, the rate of excitation of upper levels continues to increase with increasing current. The sharp optimum which occurs in the output power versus input power characteristic results instead from the population of the lower laser level by increased thermal excitation as the input power is raised. The $\left[10^{\circ} 0\right]$ state lies only about $0.17 \mathrm{eV}$ above the ground state and thus has a significant relative population (given by the Boltzmann factor exp $[-0.17 \mathrm{eV} / \mathrm{kT}]$ ) at room temperature and above. As a direct consequence, $\mathrm{CO}_{2}$ lasers usually use helium as a gas additive to remove heat from the center of the discharge to the walls by improving the thermal conductivity of the gas mixture. The discharge tubes usually use water-cooled or refrigerated walls to reduce the gas temperature further. Other gas additives are used to depopulate the lower laser level directly by collisions. Water vapor and hydrogen (which probably forms water vapor in the discharge) have both been used [86]. Xenon has also been used as an additive to sealed-off $\mathrm{CO}_{2}$ lasers to extend life, but the exact mechanism responsible for this result is not known [87].

The fast-flow $\mathrm{CO}_{2}$ laser, on the other hand, eliminates the thermal repopulation of the lower laser level by physically transporting the "spent" mixture (i.e., the mixture with a significant lower level population) out of the optical cavity by flow, and replacing it with "fresh" mixture. In such a laser, the input power per unit volume may be greatly increased without reaching an optimum as long as the flow rate is also increased. The full potential of this fast-flow laser technique has not yet been realized.

It is easy to appreciate that the usual $\mathrm{CO}_{2}$ laser mixture of $\mathrm{CO}_{2}, \mathrm{CO}, \mathrm{N}_{2}, \mathrm{He}, \mathrm{H}_{2} \mathrm{O}, \cdots$ can produce some very interesting chemistry in a discharge tube. Chemical reactions in the gas phase result in the formation of $\mathrm{CN}, \mathrm{C}_{2}$, and probably other species, so that it is not surprising that a complete understanding of the discharge processes is not yet realized. Chemical reactions between the gases and the components of the tube envelope present an even more serious problem. It is difficult to find a suitable hot cathode for this environment, thus cold cathodes are usually used. Even cold cathodes have complex chemical interactions with the discharge, resulting in the cleanup of one or more gases. The best life results to date have been obtained with small tubes (output power $<50 \mathrm{~W}$ ). Sealed-off operating lifetimes in excess of 9000 hours have been demonstrated to date [88]. These tubes usually use mixtures of $\mathrm{CO}_{2}-\mathrm{N}_{2}-$ $\mathrm{He}-\mathrm{H}_{2} \mathrm{O}-\mathrm{Xe}$, although the role of all the additives is not understood. The key problem area in the development of long-life sealed-off $\mathrm{CO}_{2}$ lasers is the understanding of the discharge chemistry. A typical life-test $\mathrm{CO}_{2}$ laser is shown in Fig. 12. This particular glass tube has one internal mirror and one GaAs Brewster's angle window.

It is possible to circumvent the problem of discharge chemistry by flowing the gas mixture slowly through the discharge tube. Dissociation products are pumped out and fresh gas of the correct mixture is continually supplied. This is the approach presently taken with all $\mathrm{CO}_{2}$ lasers of 100 - 


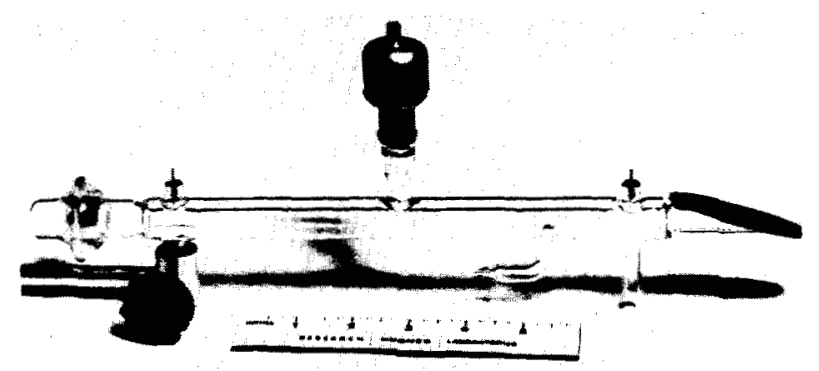

Fig. 12. Small glass sealed-off $\mathrm{CO}_{2}$ laser used in life test studies.

watt or greater output. In such continuous-flow lasers, envelope cleanliness and vacuum integrity are less important than in sealed-off tubes. As a consequence, high power $\mathrm{CO}_{2}$ lasers are generally more analogous to demountable vacuum systems than to electron tubes (as is easily seen from a close inspection of Fig. 11).

The requirement that the $\mathrm{CO}_{2}$ laser optics must pass $10.6-\mu \mathrm{m}$ radiation, often at very high flux densities also presents some fabrication problems [58]. The lowest loss materials at this wavelength are the alkali halides, with $\mathrm{NaCl}$ and $\mathrm{KCl}$ being the best. Unfortunately, these materials are hygroscopic, mechanically weak, and not easily vacuum sealed. Semiconductors such as germanium and $\mathrm{GaAs}$ have better physical properties, but they also have higher loss and suffer from thermal runaway (i.e., a positive loss temperature coefficient). The high index of refraction of germanium and GaAs makes them difficult to use for Brewster's angle windows; they are usually used as the substrate material for the output mirror in an internal mirror configuration. The development of new optical materials and sealing techniques for the $\mathrm{CO}_{2}$ laser is a challenging area. Recently, loss figures at least a factor of 2 lower than the best GaAs were reported for single-crystal CdTe [89].

Although sealed-off high-reliability $\mathrm{CO}_{2}$ lasers are not yet readily available for optical communications sources, several development programs are now underway. Metalceramic discharge tubes and versions for space application are currently being built. The ease of obtaining single-frequency output, coupled with the possibility of using a power amplifier to obtain still more power without loss of coherence makes the $\mathrm{CO}_{2}$ laser most attractive for general communications applications. The high efficiency (20 percent) obtained in even smaller lasers and the lower quantum noise $(h \nu B)$ encountered at $10.6 \mu \mathrm{m}$ make the $\mathrm{CO}_{2}$ laser particularly attractive for space communications.

\section{SEMCONDUCtor LASERS}

The very high population inversion obtainable in semiconductors results in a large gain. In fact, the gain can be so large that the path length for the stimulated radiation need not be long and the ends of the cavity need not be highly reflecting.

Thus, with cavity lengths of only a few hundred microns, semiconductor lasers are extremely small.

In contrast to other types of lasers where the transitions occur between discrete states of excited atoms, in semiconductor lasers, the transitions involve sets of banded states.
The banding of states results from the close packing of activated atoms. Hence a high density of population inversion is required to reach threshold conditions.

\section{A. Pumping of Semiconductor Lasers}

Population inversion in semiconductor lasers can be obtained either optically or electrically. Optical excitation or photoluminescence is achieved by irradiating the semiconductor with photons that have sufficient energy to produce electron-hole pairs across the energy gap [90]. However, a high intensity of lower energy photons can also be used to generate electron-hole pairs in which case two photons [91] are absorbed to complete the transition across the gap. The main advantage of optical pumping is that it can be used with materials in which $p-n$ junctions cannot be made, for example, in CdS or CdSe.

Electron beam excitation is also suitable for pumping wide gap semiconductors in which $\mathrm{p}-\mathrm{n}$ junctions cannot be fabricated. The electron beam can be easily moved, focused, and modulated.

The most practical mode of exciting a semiconductor laser is by injection at the $p-n$ junction. In injection lasers, the electrical energy is converted directly into coherent radiation making the injection laser the most efficient type of laser. Injection is obtained by forward biasing the p-n junction; in this case the input impedance of the diode is very low, thus making the use of stripline circuitry a convenient technique.

Another means of excitation of semiconductor lasers is by impact ionization of electron-hole pairs of avalanche breakdown [92]. This breakdown occurs when a field $\left(>2 \times 10^{5} \mathrm{~V} / \mathrm{cm}\right)$ is applied across a homogeneous semiconductor comprising no $\mathrm{p}-\mathrm{n}$ junction. The breakdown mode of operation is equivalent to that of gas lasers but results in a much denser plasma. Because of power dissipation, bulk excitation is obtainable in low duty cycle pulses and does not appear at present to be useful for optical communication. However, the advantage of low beam dispersion that may be anticipated in the bulk laser may spur further effort in this mode of pumping.

As we shall see later, the most practical modes of pumping semiconductor lasers are by injection, either directly into the material or by pumping optically one semiconductor with the output of an injection laser made of a different semiconductor.

\section{B. Performance of Semiconductor Lasers}

As shown in Table II the range of wavelengths achieved by semiconductor lasers extends from 0.33 to $31 \mu$. In certain alloys between compounds, which are miscible in all proportions, the laser wavelength is adjustable by the composition of the alloy.

The radiative recombination usually occurs in a very thin layer of the semiconductor, approximately equal to a minority carrier diffusion length if no special confinement methods are used. Thus, in injection lasers, the active region is about $1-2 \mu$ thick at the junction. In lasers pumped 
TABLE II

SPECtRal Range COVERED by SeMiconductor LASERS

\begin{tabular}{|c|c|c|c|c|c|c|}
\hline & $\lambda(\mu)$ & $h v(\mathrm{eV})$ & \multicolumn{4}{|c|}{ Excitation* } \\
\hline Zns & 0.33 & 3.8 & 0 & $E$ & & \\
\hline $\mathrm{ZnO}$ & 0.37 & 3.4 & & $E$ & & \\
\hline $\mathrm{Zn}_{1-x} \mathrm{Cd}_{x} \mathrm{~S}$ & $0.49-0.32$ & $2.5-3.82$ & $o$ & & & \\
\hline $\mathrm{ZnSe}$ & 0.46 & 2.7 & & $E$ & & \\
\hline$\mp \mathrm{CdS}$ & 0.49 & 2.5 & $O$ & $E$ & & \\
\hline $\mathrm{ZnTe}$ & 0.53 & 2.3 & & $E$ & & \\
\hline $\mathrm{GaSe}$ & 0.59 & 2.1 & & $E$ & & \\
\hline $\mathrm{CdSe}_{1-x} \mathrm{~S}_{x}$ & $0.49-0.68$ & $2.5-1.8$ & $o$ & $E^{\dagger}$ & & \\
\hline $\mathrm{CdSe}_{0.95} \mathrm{~S}_{0.05}$ & 0.675 & 1.8 & & $E$ & & \\
\hline $\mathrm{CdSe}$ & 0.675 & 1.8 & 0 & $E$ & & \\
\hline${ }^{*} \mathrm{Al}_{1-x} \mathrm{Ga}_{x} \mathrm{As}$ & $0.63-0.90$ & $2.0-1.4$ & & & $I$ & \\
\hline$\neq \mathrm{GaAs}_{1-x} \mathrm{P}_{x}$ & $0.61-0.90$ & $2.0-1.4$ & & $E$ & $I$ & \\
\hline $\mathrm{CdTe}$ & 0.785 & 1.6 & & $E$ & & \\
\hline$\ddagger \mathrm{GaAs}$ & $0.83-0.91^{* *}$ & $1.50-1.38$ & $O$ & $E$ & $I$ & $A$ \\
\hline InP & 0.91 & 1.36 & & & $I$ & $A$ \\
\hline $\mathrm{GaAs}_{1-x} \mathrm{Sb}_{x}$ & $0.9-1.5$ & $1.4-0.83$ & & & $I$ & \\
\hline $\mathrm{CdSnP}_{2}$ & 1.01 & 1.25 & & $E$ & & \\
\hline $\operatorname{In} A s_{1-x} P_{x}$ & $0.9-3.2$ & $1.4-3.9$ & & & $I$ & \\
\hline $\operatorname{InAs}_{0.94} \mathrm{P}_{0.06}$ & 0.942 & 1.32 & & & $I$ & \\
\hline $\mathrm{InAs}_{0.51} \mathrm{P}_{0.49}$ & 1.6 & 0.78 & & & $I$ & \\
\hline $\mathrm{GaSb}$ & 1.55 & 0.80 & & $E$ & $I$ & \\
\hline $\mathrm{In}_{1-x} \mathrm{Ga}_{x} \mathrm{As}$ & $0.85-3.1$ & $1.45-3.1$ & & & $I$ & \\
\hline $\mathrm{In}_{0.65} \mathrm{Ga}_{0.35} \mathrm{As}$ & 1.77 & 0.70 & & & $I$ & \\
\hline $\mathrm{In}_{0.75} \mathrm{Ga}_{0.25} \mathrm{As}$ & 2.07 & 0.60 & & & $I$ & \\
\hline $\mathrm{Cd}_{3} \mathrm{P}_{2}$ & 2.1 & 0.58 & $O$ & & & \\
\hline InAs & 3.1 & 0.39 & $O$ & $E$ & $I$ & \\
\hline $\mathrm{InAs}_{1-x} \mathrm{Sb}_{x}$ & $3.1-5.4$ & $0.39-0.23$ & & & $I$ & \\
\hline $\operatorname{InAs} \mathrm{As}_{0.98} \mathrm{Sb}_{0.02}$ & 3.19 & 0.39 & & & $I$ & \\
\hline $\mathrm{Cd}_{1-x} \mathrm{Hg}_{x} \mathrm{Te}$ & $3-15$ & $0.41-0.08$ & $O$ & $E$ & & \\
\hline $\mathrm{Cd}_{0.32} \mathrm{Hg}_{0.68} \mathrm{Te}$ & 3.8 & 0.33 & $O$ & & & \\
\hline $\mathrm{Te}$ & 3.72 & 0.334 & & $E$ & & \\
\hline $\mathrm{PbS}$ & 4.3 & 0.29 & & $E$ & & \\
\hline InSb & 5.2 & 0.236 & $O$ & $E$ & $I$ & $A$ \\
\hline $\mathrm{PbTe}$ & 6.5 & 0.19 & & $E$ & $I$ & \\
\hline $\mathrm{PbS}_{1-x} \mathrm{Se}_{x}$ & 3.98 .5 & $0.32-0.146$ & & $E$ & $I$ & \\
\hline $\mathrm{PbSe}$ & 8.5 & 0.146 & & $E$ & $I$ & \\
\hline $\mathrm{Pb}_{1-x} \mathrm{Sn}_{x} \mathrm{Te}$ & $6-28$ & $0.209-0.045$ & & & $I$ & \\
\hline $\mathrm{PbSnSe}$ & $8-31.2$ & $0.155-0.040$ & & & $I$ & \\
\hline
\end{tabular}

${ }^{*} A$ Avalanche breakdown.

$O$ Optical pumping.

$E$ Electron beam pumping.

$I$ Injection

$\dagger$ Boldface indicates possible mode of excitation.

** Depending on temperature and doping.

$\ddagger$ Pulsed operation at room temperature.

optically with nonpenetrating radiation the active region is also one diffusion length thick. With penetrating radiation, a larger volume of material is excited. The electron beam whose penetration is voltage dependent can also be used to excite a greater volume of material than feasible with injection lasers.

Where the active region is narrow, the stimulated radiation suffers considerable diffraction resulting in a divergent coherent beam. The divergence of the beam can be reduced considerably by special structures providing a large aperture to the emerging radiation. Thus, with the radiating mirror structure of Fig. 13 where an electron beam pumps a circular area of a GaAs wafer coupled to an external cavity, the beam divergence is about 20 minutes [93]. Another structure consists of a multilayered arrangement of serially connected $\mathbf{p}-\mathbf{n}-\mathbf{p}-\mathbf{n} \cdots$ regions where the spacing between adjacent junctions is so small $(\sim 1 \mu)$ that every

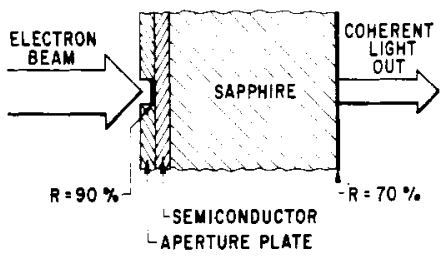

Fig. 13. Structure of electron beam excited laser in the "radiating mirror" configuration (after Basov et al. [91].

alternate $p-n$ junction which is forward biased sees the fringing electromagnetic fields of the adjacent emitting junctions [94]. This fringing field induces a common coherence or phase locking and the radiation now can escape through an effectively larger aperture.

Although special structures can be devised to minimize beam divergence, the semiconductor laser suffers from more dispersion than other types of lasers-a limitation in some applications of optical communication.

The power levels achievable with semiconductor lasers depend on permissible operating conditions. At low temperature, several watts of $\mathrm{CW}$ coherent radiation can be obtained. [95]. But at room temperature, because of large internal power dissipation (the threshold current increases exponentially with temperature [96]), the laser must be energized in pulses. Peak powers of 50 watts can be readily obtained. With a conservative repetition rate the average power is of the order of tens of milliwatts.

The optical system can be simplified with the use of the maximum possible optical density at the laser facet. However, the maximum peak power obtainable from a laser of a given cavity width is limited by the onset of catastrophic damage [97]. The flux density at which damage occurs depends on the pulsewidth, the degree of optical confinement in the laser active region, and to some extent on the temperature. At room temperature, the flux density at which castastrophic damage occurs is typically between 400 and $800 \mathrm{~W} / \mathrm{cm},{ }^{2}$ depending on the details of the fabrication process for a pulsewidth of about 100 to 200 ns. Higher peak powers are attainable with shorter pulses [97]. Recently, the use of a surface coating has raised the threshold or catastrophic damage to higher values than previously possible, thus progress in this area continues to be made [98]. A second degradation mechanism, denoted "gradual," is a function of the current density [99]. The degradation rate for a given current density depends critically on the method of laser fabrication. In particular, it appears that imperfections in the active region increase the degradation rate [99]. With current densities of $10^{4} \mathrm{~A} / \mathrm{cm}^{2}$ or less, the gradual degradation has been shown to be negligible in diffused diodes operated continuously [100].

At high power levels, the output of semiconductor lasers is multimode and can occur at several wavelengths simultaneously. Yet, pure modes can also be obtained at lower power, by resorting to special narrow structures [101]. A pure mode at high power can be produced by coupling a semiconductor laser oscillator operating as a pure mode generator to another semiconductor diode operating as a 


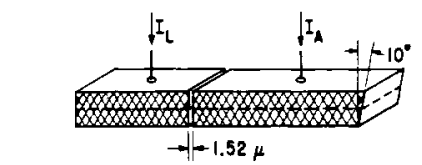

(a)

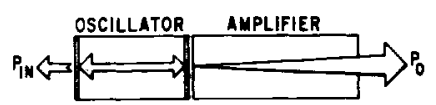

(b)

Fig. 14. Low-noise oscillator-amplifier pair. The double lines in diagram (b) represent Fabry-Perot facets (after Kosonocky and Cornely [102]).

single-pass light amplifier, Fig. 14 -[102]. When several modes can propagate simultaneously, they can interact to produce a lower beat frequency. The most remarkable mode interaction is that resulting from second-order beats [103]; because the index of refraction of semiconductors is not constant with $\lambda$ (dispersion), adjacent modes are not equally spaced. Hence, frequency mixing between two pairs of adjacent modes results in two different beat frequencies; these in turn can be mixed to generate a second-order beat which occurs at about $1 \mathrm{GHz}$ [104].

\section{Modulation and Tuning}

We have previously seen that the operating frequency of a semiconductor laser can be selected by the choice of material and in many cases adjusted by employing a suitable composition of alloy between two miscible semiconductors. Further fine tuning of the operating frequency can be obtained by adjusting the temperature (the energy gap of most semiconductors and therefore the energy of emitted photons varies at the rate of approximately $-5 \times 10^{-4} \mathrm{eV} /{ }^{\circ} \mathrm{K}$ ). Hydrostatic [105] or uniaxial pressure [106] can also be used to obtain frequency changes. Note that for injection lasers made with lead-salt semiconductors the emission energy shifts to lower values with increasing pressure [107]. A magnetic field breaks the bands into Landau levels which drift further apart with increasing field, thus shifting the emitted frequency as well [108]. A considerable tuning range is also obtainable by controlling the $Q$ of the cavity. Thus, the Fabry-Perot facets can be slightly etched or coated with a lower reflectivity layer. This requires a higher threshold or higher population inversion which pushes the quasi-Fermi levels deeper into the bands with the consequence that photons of higher energy are generated [109]. The $Q$ of the cavity can also be modulated by breaking up the active region into two separately biased sections. One of these sections can be made lossy by a reduced forward bias and even more effectively by a reverse bias [110].

The electronic control of losses in a two-section laser is an effective means of modulating the laser. The injection laser can be readily amplitude modulated up to extremely high frequencies by direct modulation of the current through the junction (up to $46 \mathrm{GHz}$ have been demonstrated [111]). When the modulation is done at high frequencies, care must be taken to design the cavity such that the mode spacing corresponds to the desired modulation frequency (the modulation is equivalent to alternating the generation of coherent radiation between two allowed cavity modes).

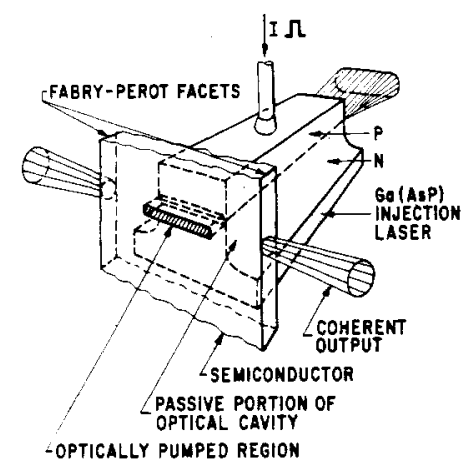

Fig. 15. Optical pumping of one semiconductor by injection laser (after Johnson and Holonyak, Jr. [90]).

Under pulsed conditions (this is how injection lasers are operated at room temperature), modulation by varying the pulse position or the pulse length are very practical methods [112]. Note that an injection laser can be used to pump optically another semiconductor of lower energy gap, Fig. 15 [90]. Then all the modulation schemes of the injection laser affect correspondingly the output of the optically pumped material.

\section{Future Prospects}

Recent breakthroughs in material technology have permitted great improvement in the performance of injection lasers. The ability to make heterotransitions between the wider gap $\mathrm{Ga}_{1-x} \mathrm{Al}_{x} \mathrm{As}$ and $\mathrm{GaAs}$ has led to the close-confinement structure [113]-[115] which constrains both the inverted population (by virtue of internal barriers to carriers) and the radiation (by producing a discontinuity in refractive index at the edge of the active region). Carrier confinement results in higher gain; radiation confinement results in lower losses since the wider gap material is less absorbing than the $\mathrm{p}^{+}$region of earlier injection lasers. The close-confinement structure has resulted in injection lasers with substantially lower threshold current densities. Threshold current densities of $10000 \mathrm{~A} / \mathrm{cm}^{2}$ are routinely obtained, as compared to typical values of $40000-60000$ in previous $\mathrm{GaAs}$ lasers. This is the trend needed to eventually realize a room temperature $\mathrm{CW}$ injection laser. Further progress in understanding and controlling diode degradation will result in improved reliability.

Progress in doping II-VI semiconductors may succeed in generating $p-n$ junctions in these compounds and thus broaden the spectral range accessible to injection lasers.

Finally, it must be pointed out that optical communication is also feasible with incoherent light sources, provided that they lend themselves readily to modulation. Thus, electroluminescent diodes, which are the most efficient and the fastest light sources, are well indicated for optical communication

\section{SOlid-STATE Ion LASERS}

Optically pumped crystalline lasers have been operated utilizing transition metal ions $\left(\mathrm{Cr}^{3+}, \mathrm{Ni}^{2+}\right.$, and $\left.\mathrm{Co}^{2+}\right)$, rare earth ions $\left(\mathrm{Nd}^{3+}, \mathrm{Pr}^{3+}, \mathrm{Er}^{3+}, \mathrm{Ho}^{3+}, \mathrm{Er}^{3+}, \mathrm{Tm}^{3+}\right.$, $\mathrm{Yb}^{3+}, \mathrm{Yb}^{2+}, \mathrm{Sm}^{2+}$, and $\left.\mathrm{Dy}^{2+}\right)$, and the actinide ion 
$\left(\mathrm{U}^{3+}\right)$ in various host lattices. Laser action in these solidstate ion systems has been reviewed by Johnson [7b], Kiss and Pressley [7a], and Goodwin and Heavens [5c]. Room temperature operation of a continuously pumped solid-state laser was first reported in 1962 by Johnson et al. [116] using $\mathrm{Nd}^{3+}$ in $\mathrm{CaWO}_{4}$. Since then $\mathrm{CW}$ room temperature operation has been reported for $\mathrm{Cr}^{3+}$ in $\mathrm{Al}_{2} \mathrm{O}_{3}$ (ruby) [117], [118] and $\mathrm{Nd}^{3+}$ in glass [119], $\mathrm{Y}_{3} \mathrm{Al}_{5} \mathrm{O}_{12}$ (YAlG) [120], $\mathrm{CaMO}_{4}$ [121], $\mathrm{Ca}_{5}\left(\mathrm{PO}_{4}\right)_{3} \mathrm{~F}$ [122], $\mathrm{YAlO}_{3}$ [123], and $\mathrm{La}_{2} \mathrm{O}_{2} \mathrm{~S}$ [124]. Of these, the most highly developed continuous solid-state ion laser is Nd:YAlG. Because of its wide range of operational capabilities, it is competitive with the three prominent gas lasers mentioned in Section IV. Our discussion of $\mathrm{CW}$ solid-state ion systems will be divided into three subsections, i.e., ruby, Nd:YAlG, and other $\mathrm{Nd}^{3+}$ systems.

\section{A. Ruby}

Historically, $\mathrm{Cr}^{3+}$ in $\mathrm{Al}_{2} \mathrm{O}_{3}$ (ruby) is of great significance because it was employed by Maiman [2] in the first laser. The ruby laser is a three-level system in which the ground state is the terminal level. Room temperature laser oscillations were reported in ruby almost simultaneously by Evtuhov and Neeland [117] and by Röss [118]. In each case high pressure mercury arc lamps were employed for pumping. Evtuhov and Neeland employed an elliptical cylinder pump cavity; Röss employed an elliptical exfocal cavity. Detailed studies of $\mathrm{CW}$ ruby oscillators are found in [125]-[127]. Using a 2-mm diameter 7.5-cm long ruby rod (0.5-percent $\mathrm{Cr}^{3+}$ concentration), the maximum $\mathrm{CW}$ output was 2.37 watts at an input of $5.08 \mathrm{~kW}$ [126]. Substantial modulation of the output was observed, and preliminary efforts to eliminate it have been unsuccessful. A second serious liability of the $\mathrm{CW}$ ruby laser is the short pump lamp life ( $\sim 25$ hours) at input power levels required to produce outputs of $\sim 1$ watt. Although the visible output of ruby and the high peak powers obtainable in the repetitively $Q$-switched mode makes it attractive for certain applications, its present liabilities exceed its assets for general communication applications.

\section{B. $N d: Y A l G$}

Some of the distinctive characteristics of $\mathrm{Y}_{3} \mathrm{Al}_{5} \mathrm{O}_{12}$ as a laser host, particularly for $\mathrm{Nd}^{3+}$, are a result of its relatively low photoelastic constants, its good mechanical and thermal properties, and the fact that trivalent ions such as $\mathrm{Nd}^{3+}$ can be incorporated substitutionally. The commercial availability of high-quality Nd:YAlG laser rods in lengths as long as $15 \mathrm{~cm}$ and diameters as large as $1 \mathrm{~cm}$ has provided sufficient latitude to permit the operation of lasers with $\mathrm{CW}$ outputs as high as 750 watts. Thus, the few watt average powers which might be required in most communication systems are well within the capabilities of the Nd:YAlG laser. In Nd:YAlG, oscillation can be achieved on a number of infrared transitions, the most prominent room temperature transition occurring at $1.064 \mu$. When nonlinear crystals are added internally to the optical cavity, efficient

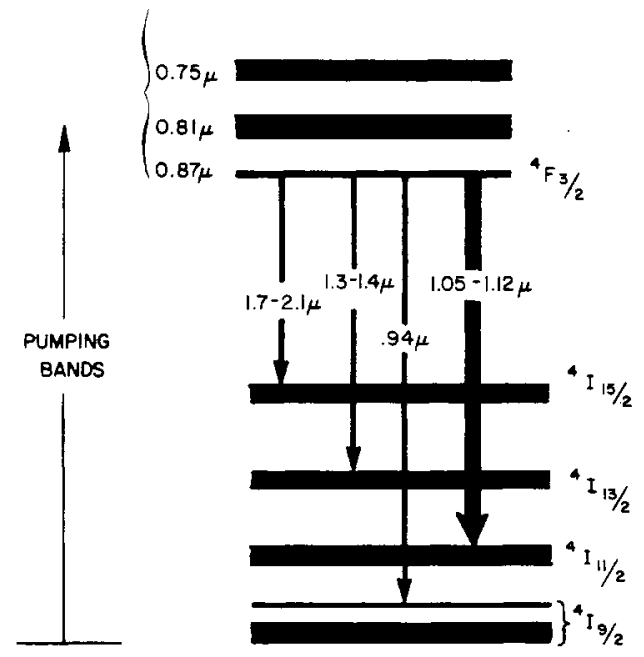

Fig. 16. Main pump bands and fluorescent transitions in Nd:YAlG. The main.laser transition is at $1.064 \mu$.

TABLE III

Main Room Temperature Transitions in Nd: YalG

\begin{tabular}{|c|c|c|c|}
\hline & $\begin{array}{l}\text { Wave- } \\
\text { length } \\
(\mu)\end{array}$ & $\begin{array}{c}\text { Peak effective } \\
\text { room temperature } \\
\text { cross section } \\
\sigma_{l}^{*}\left(10^{-19} \mathrm{~cm}^{2}\right) \dagger\end{array}$ & $\begin{array}{c}\text { Measured relative } \\
\text { room temperature } \\
\text { CW laser } \\
\text { threshold }\end{array}$ \\
\hline${ }^{4} F_{3 / 2} \rightarrow{ }^{4} I_{9 / 2}$ & $\begin{array}{l}0.939 \\
0.946\end{array}$ & $\begin{array}{l}0.81 \\
1.34\end{array}$ & \\
\hline${ }^{4} F_{3 / 2} \rightarrow{ }^{4} I_{11 / 2}$ & $\begin{array}{l}1.0520 \\
1.0551 \\
1.0615 \\
1.0641 \\
1.0682 \\
1.0738 \\
1.0779 \\
1.1055 \\
1.1122 \\
1.1161 \\
1.1225\end{array}$ & $\begin{array}{l}3.1 \\
0.20 \\
6.65 \\
8.80 \\
1.10 \\
4.00 \\
1.55 \\
0.32 \\
0.79 \\
0.77 \\
0.72\end{array}$ & $\begin{array}{l}2.08 \\
1.15 \\
1.00 \\
1.22 \\
\\
2.17 \\
2.26 \\
2.36\end{array}$ \\
\hline${ }^{4} F_{3 / 2} \rightarrow{ }^{4} I_{13 / 2}$ & $\begin{array}{l}1.319 \\
1.335 \\
1.338 \\
1.342 \\
1.353 \\
1.357\end{array}$ & $\begin{array}{l}1.50 \\
0.92 \\
1.50 \\
0.63 \\
0.35 \\
0.88\end{array}$ & $\begin{array}{l}1.60 \\
2.17\end{array}$ \\
\hline
\end{tabular}

$\dagger \sigma_{l}^{*}=\sigma_{l}\left(n_{l} / n_{u}\right)$ and $\sigma_{u}^{*}=\sigma_{u}$ where $n_{u}$ and $n_{l}$ are the upper and lower ${ }^{4} F_{3 / 2}$ stark level populations at room temperature and the $\sigma$ are the true cross sections. The peak fluorescent intensity of an individual transition at room temperature is proportion to $\sigma^{*} n_{u}$.

second-harmonic conversion of a number of the laser transitions to the visible can be achieved. The availability of $\mathrm{CW}$ outputs at several wavelengths in the visible and infrared and the possibility of operating it continuously pumped, repetitively pulsed (mode locked, cavity dumped, or $Q$-switched) make it a versatile source for communications.

1) Spectral Properties: The Nd: YAlG laser is a fourlevel system as depicted in Fig. 16. Of the main pump bands shown, the $0.81 \mu$ and $0.75 \mu$ are the strongest. The upper laser level $4 F_{3 / 2}$, has a fluorescent efficiency greater than 


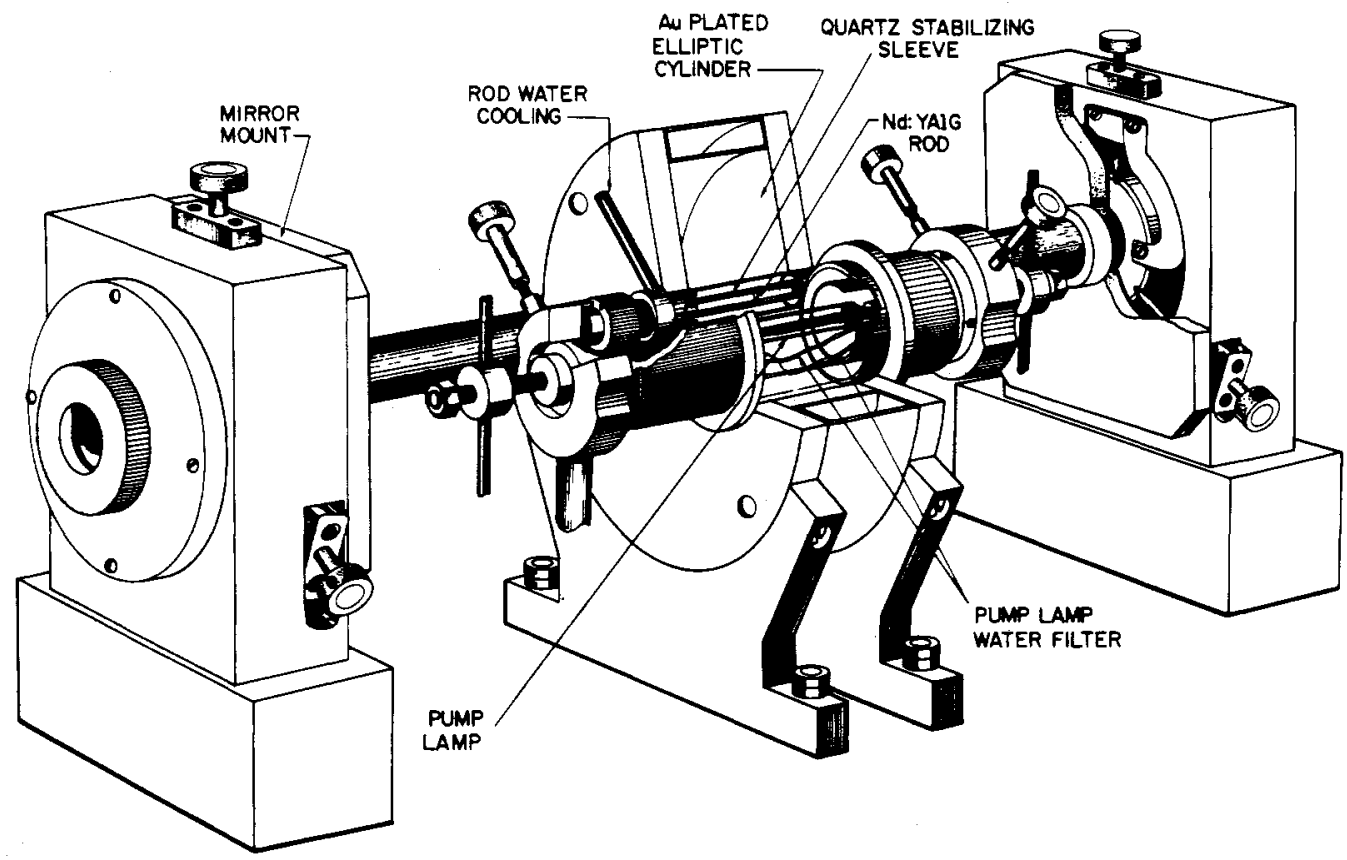

Fig. 17. Diagram of W-I pumped Nd:YAlG laser.

99.5 percent [128] and a radiative lifetime of $230 \mu$ s [120]. The branching ratio of emission from $4 F_{3 / 2}$ is as follows [129]:

$$
\begin{aligned}
& { }^{4} F_{3 / 2} \rightarrow{ }^{4} I_{9 / 2} 0.25 \\
& { }^{4} F_{3 / 2} \rightarrow{ }^{4} I_{11 / 2} 0.60 \\
& { }^{4} F_{3 / 2} \rightarrow{ }^{4} I_{13 / 2} 0.14 \\
& { }^{4} F_{3 / 2} \rightarrow{ }^{4} I_{15 / 2}<0.01 .
\end{aligned}
$$

The effective cross section [129], [130] at room temperature of the prominent transitions and the measured relative thresholds of those transitions on which room temperature $\mathrm{CW}$ oscillation has been observed [120], [131] are given in Table III. At room temperature the main $1.06-\mu$ line in $\mathrm{Nd}$ :YAlG is primarily homogeneously broadened by thermally activated lattice vibrations [120b]. The room temperature linewidth is $6.0 \mathrm{~cm}^{-1}$ or $180 \mathrm{GHz}$. The residual width at very low temperatures $\left(T<4^{\circ} \mathrm{K}\right)$ is a measure of the inhomogeneous width, which is caused by variations in the crystalline electric field from ion to ion in the lattice. The inhomogeneous width varies from crystal to crystal and is highly dependent on material quality. The narrowest width measured was $0.2 \mathrm{~cm}^{-1}$; strained crystals have linewidths as large as $1.0 \mathrm{~cm}^{-1}$ [120b].

2) Pumping Lamps and Cavities: A number of different cavities and pump lamps have been used in the operation of the Nd:YAlG laser. The original Nd:YAlG laser utilized a W-I pump lamp in an elliptical cylinder. This combination is the most highly developed, and a design utilized by one of the authors and his colleagues at Bell Telephone Laboratories is shown in Fig. 17. A packaged commercial version of a 10-watt tungsten-pumped YAlG laser system is shown in Fig. 18. W-I lamps have been developed commercially for photography and are available in a variety of

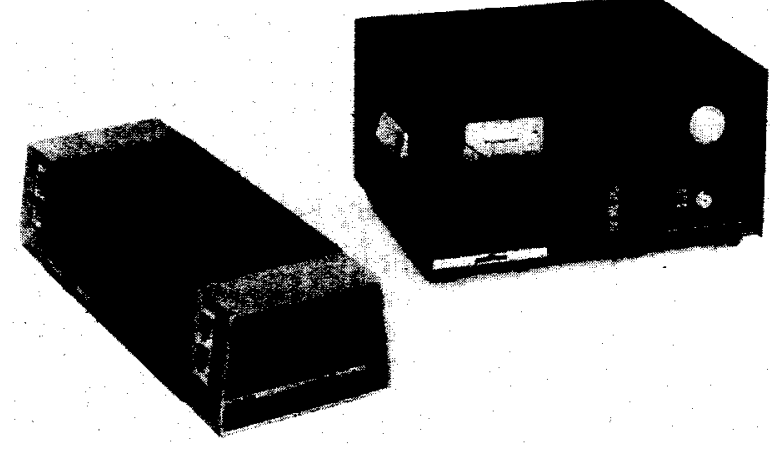

Fig. 18. Commercial, W-I pumped Nd:YAlG laser which has rated output of $\sim 10$ watts. Lamp power supply and closed cycle water cooling systems are shown on the right.

sizes. For lamps operating at a filament temperature of $3400^{\circ} \mathrm{K}$, lamp life is typically 200 hours. Lamps designed for $3000^{\circ} \mathrm{K}$ operation have a rated life of typically 2000 hours. In an operational test [132] on a 1-watt laser using a $3000^{\circ} \mathrm{K}$ lamp, a lamp life of between 3000-5000 hours was found typical. The operational life of the Nd:YAlG laser is generally determined by lamp life and the life of ancillary equipment such as the lamp power supply, etc. For moderate power (1 to 25 watts) Nd:YAlG laser applications, the W-I lamp has proven to be the most convenient and long lived of all presently available lamps.

In addition to the elliptical cylinder, spherical [133] and ellipsoidal [134] pump cavities have been used with the Nd:YAlG laser. These cavities are more costly and inconvenient to use than the elliptical cylinder. For high power lasers which use large diameter rods ( $>5 \mathrm{~mm}$ ), two pump lamps are often employed in a double-elliptical cylinder configuration.

High pressure rare gas arc lamps [135]-[137] have been 
TABLE IV

Characteristics OF RoOM Temperature Nd: YAlG Lasers

\begin{tabular}{|c|c|c|c|c|c|}
\hline Mode of operation & \multicolumn{4}{|c|}{$1.064 \mu$} & $0.532 \mu$ \\
\hline High power $C W$ multimode & \multicolumn{4}{|c|}{ 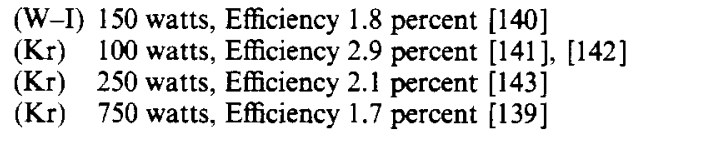 } & \\
\hline $\begin{array}{l}\text { Low power multimode } \\
\text { Single transverse mode } \\
\text { Single axial mode }\end{array}$ & \multicolumn{4}{|c|}{ (W-I) $10-15$ watts, Efficiency $0.66-1.0$ percent [138], [149] } & 4 watts [161] \\
\hline $\begin{array}{l}\text { Repetitively } Q \text {-switched } \\
\text { Peak power } \\
\text { Average power } \\
\text { Repetition rate (pps) } \\
\text { Typical pulsewidth }(\mu \mathrm{s})\end{array}$ & $\begin{array}{ll}10^{3} P_{\mathrm{CW}} & 3 \times \\
0.25 P_{\mathrm{CW}} & 0.73 \\
10^{3} & 4 \times \\
0.25 & 0.61\end{array}$ & $\begin{array}{l}0^{2} P_{\mathrm{CW}} \\
P_{\mathrm{CW}} \\
0^{3}\end{array}$ & $\begin{array}{l}10^{1} P_{\mathrm{CH}} \\
P_{\mathrm{CW}} \\
5 \times 10^{4} \\
2.0\end{array}$ & {$[52],[158]$} & $\sim$ same as $1.064 \mu$ \\
\hline $\begin{array}{l}\text { Repetitive cavity dumped } \\
\text { Peak power } \\
\text { Average power } \\
\text { Repetition rate (pps) } \\
\text { Typical pulsewidth (ns) }\end{array}$ & \multicolumn{4}{|l|}{$\begin{array}{l}1-2 \times 10^{2} P_{\mathrm{CW}} \\
P_{\mathrm{CW}} \\
10^{5} \\
(50-100)\end{array}$} & \\
\hline $\begin{array}{l}\text { Mode locked } \\
\text { Average power } \\
\text { Peak power } \\
\text { Repetition rate (pps) } \\
\text { Pulsewidth (ps) }\end{array}$ & $\begin{array}{l}P_{\mathrm{CW}} \\
\sim 3 \times 10^{2} P_{\mathrm{CW}} \\
10^{8} \\
30\end{array}$ & $\begin{array}{l}P_{\mathrm{CW}} \\
\sim 3 \times \\
10^{9} \\
30\end{array}$ & $10^{1} P_{\mathrm{CW}}$ & {$[40],[154]$} & $\sim$ same as $1.064 \mu$ \\
\hline
\end{tabular}

used to pump Nd:YAlG. The results of comparisons of the efficiency of the rare gas lamps relative to $\mathrm{W}-\mathrm{I}$ are listed below:

$\begin{array}{llll}\mathrm{Kr} & \mathrm{Xe} & \mathrm{Ar} & \\ 1.2 & 0.77 & 0.58 & {[135]} \\ 3.1 & 1.6 & & {[136]} \\ 0.95 & 0.73 & & {[137] .}\end{array}$

The measurements reported in [135] and [137] are in relatively good agreement and were carefully conducted with lamps with almost identical illuminated lengths and diameters, and under identical lamp loading conditions. The advantage of the $\mathrm{Kr}$-arc lamp from a pumping point of view is the fact that it can be operated at a higher loading level than W-I; its disadvantage is its generally shorter life. When lamp life is not a major consideration, $\mathrm{Kr}$ is the choice for high power operation.

$\mathrm{K}-\mathrm{Hg}$ discharges have also been utilized and compared with the W-I lamp [138]. When similar size $\mathrm{K}-\mathrm{Hg}$ and W-I lamps were used in the same laser and operated at their maximum input powers the laser output power was almost identical; however, the efficiency with $\mathrm{K}-\mathrm{Hg}$ was 2.4 times higher than with W-I. It is necessary to point out that in these experiments the $\mathrm{K}-\mathrm{Hg}$ lamps could not be run with dc excitation because of cataphoresis; this, the fact that the lamp is very expensive, and the fact that it is short lived make it impractical for most applications at this time.

3) $C W$ Performance Characteristics: The very high power capabilities of $\mathrm{Nd}$ :YAlG have been convincingly demonstrated by a recent announcement [139] of the achievement of 750 watts of $\mathrm{CW}$ output at 1.7-percent efficiency from a multiple-head $\mathrm{Kr}$-pumped system. Previous work [140]-[143] on lasers operating with outputs above 100 watts is summarized in Table IV. In all cases the output is highly multitransverse moded. At these high pump power levels the effects of thermally induced lens and birefringence effects [144]-[147] make efficient operation in the $\mathrm{TEM}_{00}$ mode difficult. Methods to compensate for these thermal effects have been proposed [148]; however, their effective implementation has not been carried out.

Since, in most communication systems several watts of average power is probably adequate, our further discussion will be devoted to the performance of Nd:YAlG lasers with outputs of 1-15 watts. The most efficient [138], [149] performance in this power range using W-I is 1 percent. Using the pumping geometry shown in Fig. 17 with a 3.8$\mathrm{mm}$ diameter $6-\mathrm{cm}$ long $\mathrm{Nd}: \mathrm{YAlG}$ rod (1 atomic percent $\mathrm{Nd}^{3+}$ ) of the highest quality and a commercial (1500 $\mathrm{W}-Q / 1500 / \mathrm{T} 4 / 14 \mathrm{CL}$ ) W-I lamp, an output of 15 watts at an overall efficiency of 1 percent was achieved [149]. With more typical high-quality Nd:YAlG rods the output powers observed were 10 watts at an efficiency of 0.66 percent. The lamp operates at $3200^{\circ} \mathrm{K}$ and is rated by the manufacturer (GE or Sylvania) at 500 hours. When used in the laser, typical lamp life is also 500 hours; several lamps have operated as long as 1500 hours. There are two important design features incorporated into the pumping cavity in Fig. 17 which permit good operational performance. The first is the use of the water filter around the lamp which reduces the heat load to the rod cooling system. Typically, the laser rod is cooled by water flowing around it. Because of the convective nature of this type of cooling, the rod is 
cooled unevenly in time and space. The quartz sleeve [150], in Fig. 17, dampens these thermal variations, and its use is particularly important for stable operation. In multitransverse mode operation amplitude fluctuations are less than 0.1 percent. Using a cavity which restricts operation to the $\mathrm{TEM}_{00}$ mode, a single transverse mode power was obtained which was 40 percent of the maximum multimode power. The amplitude fluctuations in $\mathrm{TEM}_{\mathbf{0 0}}$ mode operation are less than 1 percent.

4) Single Frequency Operation: The width of the frequency spectrum of a typical multiaxial-mode Nd:YAlG lasers is $15-30 \mathrm{GHz}$. Single frequency operation has been attained [35], [151] by using an intracavity etalon, as described in Section III-B. The single frequency output of a $\mathrm{Nd}$ : YAlG laser can be tuned over $\sim 100 \mathrm{GHz}$ by adjusting the tilt angle or temperature of the etalon [152]. Employing a birefringent etalon to provide both axial-mode selection and a frequency discriminant, a stabilized single frequency laser has been operated with frequency fluctuations less than 2 percent [35]. In this experiment the single frequency power was 40 percent of the multimode power. Single frequency operation at the full multimode power has been demonstrated by eliminating spatial hole burning [153].

5) Continuously Pumped, Repetitively Pulsed Operation: All of the techniques discussed in Section II-C have been applied to the Nd:YAlG laser.

Mode locking has been accomplished by using both loss and phase modulation [40], [154]. Pulses with widths of 30$50 \mathrm{ps}$ have been generated at rates as high as $5 \times 10^{8}$ pps. Pulse envelope phase instabilities have been encountered in both $\mathrm{He}-\mathrm{Ne}$ [155], [156] and Nd:YAlG [156] using a phase modulator for mode locking. A solution to these problems has been reported [157], and a stabilized mode locked Nd:YAlG has been operated which is suitable for use in an optical PCM system.

The relatively long lifetime ( $230 \mu \mathrm{s})$ of the upper laser level permits the generation of high peak powers by repetitive $Q$ switching [52]. At repetition frequencies above the reciprocal of the lifetime $(>4 \mathrm{kHz})$, the average power of the repetitively $Q$-switched laser approaches the output power obtainable in normal $\mathrm{CW}$ operation. At repetition rates $\leq 2 \mathrm{kHz}$ the pulses generated have a peak power $\sim 10^{3}$ times the laser's normal $\mathrm{CW}$ power. At higher repetition frequencies the peak power is approximately proportional to the reciprocal of the square of the repetition frequency. Stable operation has been achieved at repetition frequencies up to $50 \mathrm{kHz}$ [55], [158]. The finite buildup time of the field inside the laser cavity and the time required to repump the inversion sets an upper limit to the repetition rates at which good pulse-to-pulse amplitude stability is obtainable. A detailed experimental and theoretical study of repetitive $Q$ switching in Nd:YAlG is found in [158].

Pulsed operation at repetition frequencies from approximately $100 \mathrm{kHz}$ to $30 \mathrm{MHz}$ is best accomplished in $\mathrm{Nd}$ : YAlG by cavity dumping [49]. Operation over the repetition frequency range $125 \mathrm{kHz}$ to $5 \mathrm{MHz}$ has recently been reported [159]. At $125 \mathrm{kHz}$, for example, 200-watt pulses of $80 \mathrm{~ns}$ were achieved, with an average power which was equal to the normal $\mathrm{CW}$ power. The low-frequency limit of

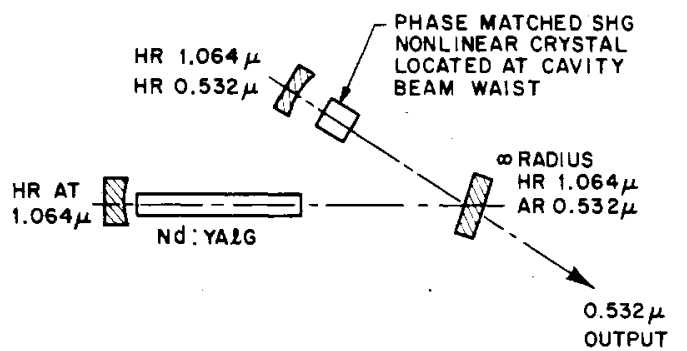

Fig. 19. Schematic diagram of a doubled Nd:YAlG laser.

stable dumping in Nd:YAlG has been discussed theoretically [49]. In Nd:YAlG cavity dumping provides efficient pulsed operation in the repetition frequency range where mode locking or stable $Q$ switching is either impractical or impossible.

6) Doubled Nd:YAlG: Visible outputs from the Nd: YAlG laser have been obtained by incorporating into the optical cavity a nonlinear material which can be phase matched for second-harmonic generation; this is schematically shown in Fig. 19. Functionally, the second harmonic crystal acts as an output coupler in a manner analogous to the transmitting mirror of a normal laser. In the normal laser the transmitting mirror couples out power at the laser frequency, whereas the nonlinear crystal inside the laser couples power at twice the frequency. It has been shown both experimentally and theoretically [27], [29] that, by properly employing a crystal of sufficient nonlinearity in the Nd:YAlG laser, it is possible to convert completely the available output at the fundamental to the harmonic. Complete conversion of the 1.064- $\mu$ output has been reported [27] using barium sodium niobate [160]. A TEM To $_{\text {mode }}$ output of 4-watts at $0.532 \mu$ has been generated [161] using a W-I pumped laser of the type described in Section VI-B 3. With barium sodium niobate it is possible to efficiently double many of the other infrared outputs in Table III. In particular $>70$ percent $\mathrm{CW}$ conversion of the $1.32-\mu$ line to $0.66-\mu$ has been obtained recently [162].

In a doubled $\mathrm{Nd}$ : YAlG source having many axial modes oscillating, amplitude fluctuations at the harmonic will occur as a result of axial mode phase fluctuations at the fundamental. To achieve a stable harmonic amplitude, some form of phase stabilization such as mode locking or FM phase locking is necessary. Also, a stable harmonic output can be achieved by operating the laser in a single axial mode.

7) Diode Pumping: A compact and simple laser can be realized by utilizing efficient incoherent electroluminescent diodes to pump Nd:YAlG. For example, lasers have been operated [163], [164] utilizing an array of $\mathrm{GaAs}_{0.87} \mathrm{P}_{0.13}$ diodes. The diodes which were employed were cooled to $77^{\circ} \mathrm{K}$ in order to shift the spectral output into the desired $0.81-\mu$ pump band region. With the diodes and rod at $77^{\circ} \mathrm{K}$, laser threshold was achieved with $300 \mathrm{~mW}$ of electrical power into the diodes [163]. An input power of 6 watts was required to reach threshold when the rod was at room temperature [164]. A schematic diagram and a working model [164] of a diode-pumped laser are shown in Figs. 20 and 21 .

Recently room temperature GaAsP diodes which emit 


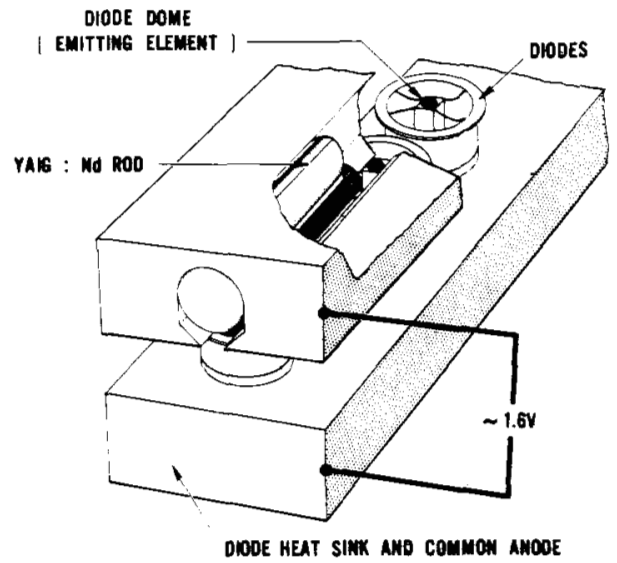

Fig. 20. Diagram of a diode-pumped Nd:YAlG laser. Diodes depicted are incoherent domed emitters such as GaAsP.

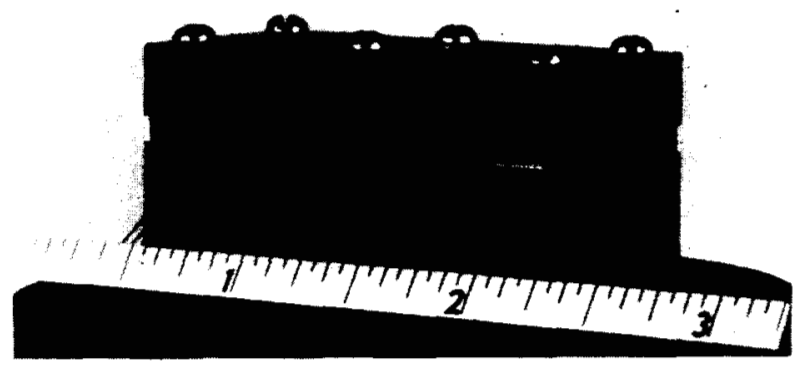

Fig. 21. Working model of diode-pumped Nd:YAlG laser. This model uses diodes which have to be cooled to $77^{\circ} \mathrm{K}$ in order to shift their spectral output into the desired $0.81-\mu$ pump band region.

at $0.81 \mu$ have been built with typical efficiencies of 4 percent and a few as high as 8 percent [165]. With such diodes a completely room temperature diode-pumped Nd:YAlG laser should be realized in the very near future. This type of compact source could be extremely useful in the planning of a practical optical communication system.

\section{Other Nd Systems}

Of the other $\mathrm{Nd}^{3+}$ systems which have operated continuously at room temperature $\mathrm{Ca}_{5}\left(\mathrm{PO}_{4}\right)_{3} \mathrm{~F}$ [122], $\mathrm{YAlO}_{3}$ [123], and $\mathrm{La}_{2} \mathrm{O}_{2} \mathrm{~S}$ [124] are most noteworthy. On the basis of spectral data they should be comparable to $\mathrm{Nd}$ : YAlG in terms of intrinsic laser performance. Thus far, however, none of the systems has actually matched the performance of $\mathrm{Nd}$ : YAlG, and further development will be required before they are practically competitive. The poor thermal conductivity of $\mathrm{Ca}_{5}\left(\mathrm{PO}_{4}\right)_{3} \mathrm{~F}$ and $\mathrm{La}_{2} \mathrm{O}_{2} \mathrm{~S}$ precludes the possibility of their utilization in high-power laser systems. $\mathrm{YAlO}_{3}$ is mechanically and thermally similar to $\mathrm{Y}_{3} \mathrm{Al}_{5} \mathrm{O}_{12}$ and, if twinning during the growth of $\mathrm{YAlO}_{3}$ can be overcome, it will be useful.

\section{Parametric Oscillators}

The observation of continuous parametric oscillation in 1968 [166], [167] demonstrated the feasibility of a coherent tunable continuous source for communications. The oscillator reported by Byer et al. [167] used $\mathrm{LiNbO}_{3}$ as the nonlinear medium. Using an Ar laser they observed a threshold of $410 \mathrm{~mW}$, an output power of $1.5 \mathrm{~mW}$, and a tuning range of $0.68 \mu$ to $0.705 \mu$ for the signal and $2.1 \mu$ to $1.9 \mu$ for the

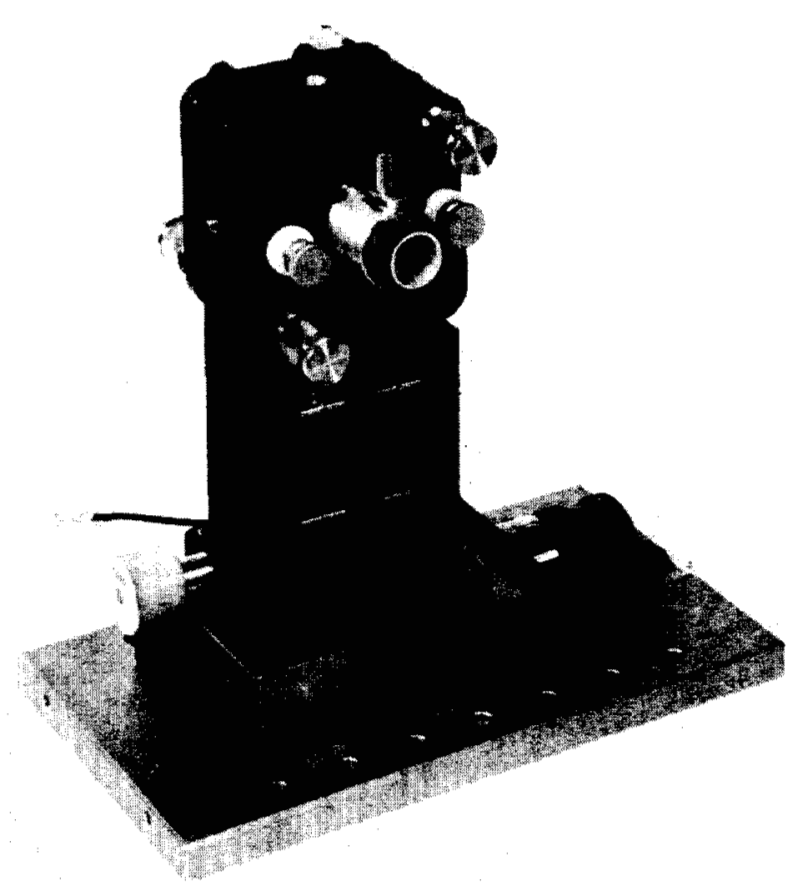

Fig. 22. Doubly resonant $\mathrm{CW}$ parametric oscillator.

idler. In the oscillator of Smith et al. [166], the nonlinear material was $\mathrm{Ba}_{2} \mathrm{NaNb}_{5} \mathrm{O}_{15}$ [160] and the pump was a $0.532-\mu$ doubled Nd:YAlG source. A threshold of 45 $\mathrm{mW}$, an output power of $3 \mathrm{~mW}$, and a tuning range from 0.98 to $1.16 \mu$ were observed. The outputs from these early continuous parametric oscillators had severe amplitude fluctuations (100 percent,) as well as oscillating bandwidths of $1-10 \mathrm{~cm}^{-1}$.

In recent parametric oscillator studies (using $\mathrm{Ba}_{2} \mathrm{NaNb}_{5} \mathrm{O}_{15}$ ) by Smith [168], the linewidth and amplitude stability have been improved by several orders of magnitude by the use of a frequency stabilized pump source which is isolated from the parametric oscillator. Using such a stabilized Ar laser $0.514-\mu$ pump, the recent results include a reduction of the threshold power to $2.8 \mathrm{~mW}$, an increase in the power output and efficiency to $45 \mathrm{~mW}$ and 30 percent, respectively, a reduction of amplitude fluctuations to $\sim 20$ percent, and single longitudinal mode operation of the oscillator. A typical doubly resonant continuous optical parametric oscillator used by Smith [168] in his studies is shown in Fig. 22.

Optical parametric oscillators are still in an embryonic stage of development and additional effort on amplitude and frequency stabilization will be required before they can be used in communications. The development of new nonlinear materials with even higher nonlinearities than $\mathrm{Ba}_{2} \mathrm{NaNb}_{5} \mathrm{O}_{15}$ could permit the operation of continuous singly resonant oscillators in which either the signal or idler but not both are resonated. Although a singly resonant oscillator has a higher pump threshold power than an equivalent doubly resonant oscillator, the singly resonant oscillator can be stabilized and tuned more easily. At present, only pulse pumped operation of singly resonant oscillators has been possible [169]-[172]. For a detailed discussion of parametric oscillators the reader is again referred to the review by Harris [24]. 


\section{SUMMARY AND CONCLUSIONS}

The favored candidates for high capacity optical communications systems are the $\mathrm{He}-\mathrm{Ne}$, argon ion, $\mathrm{Nd}$ :YAG (plain or doubled), and $\mathrm{CO}_{2}$ lasers. The choice of one or another will depend on the particular system requirements, such as location (terrestial or space), efficiency, life, etc. The existing state of the art in modulation [173] and detection [174] will also influence the choice. Of these sources, $\mathrm{He}-\mathrm{Ne}$ is by far the most highly developed and at the present the most suitable for applications where long life and average powers less than $100 \mathrm{~mW}$ are required, and where large size and low efficiencies are not major considerations. Future $\mathrm{He}-\mathrm{Ne}$ development is expected to result primarily in cost reduction.

In systems using average powers $>100 \mathrm{~mW}$, the $\mathrm{Ar}$ ion doubled Nd:YAlG, and $\mathrm{CO}_{2}$ lasers are the main contenders. There has been a greater commercial development of the Ar laser, and thus it tends to be more readily available at present. Its low efficiency ( 0.2 percent or less) is not expected to improve and major advances are expected to be in the area of extending its present life ( $<1000$ hours). The doubled Nd: YAlG laser is more efficient ( $>0.2$ percent), smaller in size, and longer lived (pump lamp life is $>1000$ hours and that of the basic laser is much greater) than the Ar laser. The problem associated with obtaining the required highquality nonlinear $\mathrm{Ba}_{2} \mathrm{NaNb}_{5} \mathrm{O}_{15}$ crystals appears to be improving, since such crystals are now being supplied routinely by suppliers such as Linde Company. Another feature of doubled Nd:YAlG is its shorter mode locked pulses which allow for higher capacity time-multiplexed PCM systems [30]. The major area of development of this source will be centered around attempts to realize a practical diode pumped $\mathrm{Nd}$ : YAlG laser.

At present, the $\mathrm{CO}_{2}$ laser is the most efficient ( 20 percent), and it has virtually unlimited power capabilities. In addition, near-ideal performance is obtained in high quantum efficiency detection, and an order of magnitude lower quantum noise $(h v B)$ is realized at $10.6 \mu \mathrm{m}$. The major drawbacks in the use of $\mathrm{CO}_{2}$ lasers in some types of communications systems are the requirement of cooled detectors at $10.6 \mu \mathrm{m}$ and the somewhat higher modulation drive power per unit bandwidth. Modulator and detector advances could greatly enhance the variety of systems using $\mathrm{CO}_{2}$ lasers in the future, although systems with bandwidths greater than $500 \mathrm{MHz}$ are possible now.

Although most of the presently developed sources are adequate for communications research, none can yet compete with respect to small size and high efficiency with microwave or baseband solid-state devices used in present day low-frequency communication systems. From the results of the work to date it appears that in the near future a continuous room temperature junction laser and/or the diode-pumped Nd:YAlG laser will change this situation. It is clear at this juncture that additional optical source development is required before an optical communications system will be economically competitive with the lower frequency systems presently being used.
Continuous room temperature operation of a double heterostructure, $\mathrm{GaAs}-\mathrm{Al}_{x} \mathrm{Ga}_{1-x} \mathrm{As}$, injection laser has been reported by I. Hayashi, M. B. Pannish, P. W. Foy, and S. Sumski in the August 1, 1970 issue of Applied Physics Letters.

Recent experiments on diode pumping of the Nd:YAlG laser have demonstrated the feasibility of obtaining room temperature $\mathrm{CW}$ operation with presently available $\mathrm{GaAs}_{1-x} P_{x}$ diodes. Using 19, 4 percent efficient, incoherent, room temperature diodes with a total power input of 7.6 watts, threshold has been obtained with the laser rod cooled to $-2.5^{\circ} \mathrm{C}$. From these measurements it is concluded that 28 room temperature diodes of the same 4-percent efficiency are needed to reach threshold when the $\mathrm{Nd}$ : YAlG rod is at room temperature $\left(20^{\circ} \mathrm{C}\right)$. The number of diodes in the above experimental array can be increased by a factor of 3 to 4 . Using 60 diodes the Nd:YAlG laser would be 2.2 times above threshold and with 80 diodes 2.9 times above threshold. This work is described in a letter which has been submitted to Applied Physics Letters by F. W. Ostermayer, Jr.

\section{ACKNOWLEDGMENT}

The authors wish to thank their colleagues at Bell Telephone Laboratories, Hughes Research Laboratories, and RCA Laboratories, whose work and constructive suggestions they have used liberally. The authors also wish to thank Hughes Electron Dynamics Division for Figs. 6 and 7; Hughes Research Laboratories for Figs. 8-12; Quantronix Corporation for Fig. 18; and F.W. Ostermayer and R. G. Smith, Bell Telephone Laboratories, for Figs. 21 and 22 , respectively.

\section{REFERENCES}

[1] A. L. Schawlow and C. H. Townes, "Infrared and optical masers," Phys. Rev., vol. 112, pp. 1940-1949, December 1958.

[2] T. H. Maiman, "Stimulated optical radiation in ruby masers," Nature, vol. 187, pp. 493-494, August 1960.

[3] A. Javan, W. R. Bennett, Jr., and D. R. Herriott, "Population inversion and continuous optical maser oscillation in a gas discharge containing a helium-neon mixture," Phys. Rev. Lett., vol. 6, pp. 106-110, February 1961.

[4] A. Yariv, Quantum Electronics. New York: Wiley, 1967, ch. 15, pp. 242-243.

[5] a) A. Yariv and J. P. Gordon, "The laser," Proc. IEEE, vol. 51, pp. 4-29, January 1963.

b) J. E. Geusic and H. E. D. Scovil, "Microwave and optical masers," Rep. Prog. Phys., vol. 28, pp. 241-327, 1964.

c) D. W. Goodwin and O. S. Heavens, "Doped crystal and gas lasers," Rep. Prog. Phys., vol. 31, pt. 2, pp. 777-859, 1968.

[6] a) A. L. Bloom, "Gas lasers," Proc. IEEE, vol. 54, pp. 1262-1276, October 1966.

b) C. K. N. Patel, "Gas lasers," in Lasers, vol. 2, A. K. Levine, Ed. New York: Dekker, 1968, pp. 1-190.

[7] a) Z. J. Kiss and R. J. Pressley, "Crystalline solid lasers," Proc. IEEE, vol. 54, pp. 1236-1248, October 1966.

b) L. F. Johnson, "Optically pumped pulsed crystal lasers other than ruby," in Lasers, vol. 1, A. K. Levine, Ed. New York: Dekker, 1966, pp. 137-180.

[8] a) M. I. Nathan, "Semiconductor lasers," Proc. IEEE, vol. 54, pp. 1276-1290, October 1966 .

b) W. P. Dumke, "The injection laser," in Lasers, vol. 1, A. K. Levine, Ed. New York: Dekker, 1968, pp. 257-293. 
[9] a) G. Birnbaum, Optical Masers. New York: Academic Press, 1964.

b) B. A. Lengyel, Introduction to Laser Physics. New York: Wiley, 1966

c) W. V. Smith and P. P. Sorokin, The Laser. New York: McGraw-Hill, 1966.

d) D. Röss, Lasers, Light Amplifiers and Oscillators. New York: Academic Press, 1969

[10] H. Kogelnik, "Modes in optical resonators," in Lasers, vol. 1, A. K. Levine, Ed. New York: Dekker, 1966, pp. 295-347.

[11] H. Kogelnik and T. Li, "Laser beams and resonators," Proc. IEEE, vol. 54, pp. 1312-1329, October 1966.

[12] a) A. G. Fox and T. Li, "Resonant modes in a maser interferometer," Bell Sys. Tech. J., vol. 40, pp. 453-488, March 1961.

b) G. D. Boyd and J. P. Gordon, "Confocal multimode resonator for millimeter through optical wavelength lasers," Bell Sys. Tech. J., vol. 40, pp. 489-508, March 1961.

c) G. D. Boyd and H. Kogelnik, "Generalized confocal resonator theory," Bell Sys. Tech. J., vol. 41, pp. 1347-1369, July 1962.

[13] M. Scully, "The quantum theory of a laser, a problem in nonequilibrium statistical mechanics," in Quantum Optics, R. J. Glauber, Ed. New York: Academic Press, 1969, pp. 586-629.

[14] H. Haken and W. Weidlich, "Quantum theory of the laser," in Quantum Optics, R. J. Glauber, Ed. New York: Academic Press, 1969 , pp. 630-679.

[15] J. P. Gordon, "Quantum theory of a simple laser," in Quantum Optics, R. J. Glauber, Ed. New York: Academic Press, 1969, pp. 741-789.

[16] R. J. Glauber, "Coherence and quantum detection," in Quantum Optics, R. J. Glauber, Ed. New York: Academic Press, 1969, pp. $15-56$.

[17] F. T. Arecchi, "Photocount distribution and field statistics," in Quantum Optics, R. J. Glauber, Ed. New York: Academic Press, 1969, pp. 57-110.

[18] Lord Rayleigh, Phil. Mag., vol. S5, 1883.

[19] W. H. Louisell, Coupled Mode and Parametric Electronics. New York: Wiley, 1960.

[20] J. Ducuing, "Nonlinear optical processes," in Quantum Optics, R. J. Glauber, Ed. New York: Academic Press, 1969, pp. 421472.

[21] Y. R. Shen, "Quantum theory of nonlinear optics," in Quantum Optics, R. J. Glauber, Ed. New York: Academic Press, 1969, pp. 473-492.

[22] J. A. Giordmaine, "Parametric optics," in Quantum Optics, R. J. Glauber, Ed. New York: Academic Press, 1969, pp. 493-519.

[23] R. W. Minck, R. W. Terhune, and C. C. Wang, "Nonlinear optics," Proc. IEEE, vol. 54, pp. 1357-1374, October 1966

[24] S. E. Harris, "Tunable optical parametric oscillators," Proc. IEEE, vol. 57, pp. 2096-2113, December 1969.

[25] R. W. Terhune and P. D. Maker, "Nonlinear optics," in Lasers, vol. 2, A. K. Levine, Ed. New York: Dekker, 1968, pp. 295-372.

[26] G. D. Boyd and D. A. Kleinman, "Parametric interaction of focused Gaussian light beams," J. Appl. Phys., vol. 39, pp. 3597-3639, July 1968.

[27] J. E. Geusic, H. J. Levinstein, S. Singh, R. G. Smith, and L. G. Van Uitert, "Continuous $0.532 \mu$ solid state sources using $\mathrm{Ba}_{2} \mathrm{NaNb}_{5} \mathrm{O}_{15}$," Appl. Phys. Lett., vol. 12, pp. 306-308, May 1, 1968 .

[28] M. W. Dowley, "Efficient CW second-harmonic generation to 2573 A," Appl. Phys. Lett., vol. 13, pp. 395-397, December 1, 1968.

[29] R. G. Smith, "Theory of intracavity optical second-harmonic generation, IEEE J. Quantum Electron., vol. QE-6, pp. 215-223, April 1970.

[30] a) T. S. Kinsel, "Wide-band optical communication system, pt. I: Time division multiplexing." Proc. IEEE, this issue, pp. 1666-1683. b) O. E. DeLange, "Wide-band optical communication system, pt. II: Frequency-division multiplexing," Proc. IEEE, this issue, pp. $1683-1690$.

[31 ] H. Kogelnik, "Modes in optical resonators," in Lasers, vol. 1, A. K. Levine, Ed. New York: Dekker, 1966, pp. 330-334.

[32] A. L. Bloom, Gas Lasers. New York: Wiley, 1968, pp. 82-85.

[33] A. G. Fox and T. Li, "Effect of gain saturation on the oscillating modes of optical masers," IEEE J. Quantum Electron., vol. QE-2, pp. 774-783, December 1966.

[34] A. L. Bloom, Gas Lasers. New York: Wiley, 1968, pp. 86-100, $122-137$.
[35] H. G. Danielmeyer, "Stabilized efficient single-frequency Nd:YAG laser,"' IEEE J. Quantum Electron., vol. QE-6, pp. 101-104, February 1970.

[36] T. G. Polanyi and I. Tobias, "The frequency stabilization of gas lasers," in Lasers, vol. 2, A. K. Levine, Ed. New York: Dekker, 1968, pp. 373-423.

[37] S. E. Harris, "Stabilization and modulation of laser oscillators by internal time-varying perturbations," Proc. IEEE, vol. 54, pp. 14011413, October 1966.

[38] L. E. Hargrove, R. L. Fork, and M. A. Pollack, "Locking of He-Ne modes induced by synchronous intracavity modulation," Appl. Phys. Lett., vol. 5, pp. 4-5, July 1964.

[39] M. H. Crowell, "Characteristics of mode-coupled lasers," IEEE J. Quantum Electron., vol. QE-1, pp. 12-20, April 1965.

[40] M. DiDomenico, J. E. Geusic, H. M. Marcos, and R. G. Smith, "Generation of ultrashort optical pulses by mode locking the YAlG:Nd lasers," Appl. Phys. Lett., vol. 8, pp. 180-182, April 1, 1966.

[41] M. DiDomenico, "Small signal analysis of internal (coupling type) modulation of lasers," J. Appl. Phys., vol. 35, pp. 2870-2876, October 1964.

[42] S. E. Harris and O. P. McDuff, "Theory of FM laser oscillation," IEEE J. Quantum Electron., vol. QE-1, pp. 245-262, September 1965.

[43] P. W. Smith, "Mode-locking of lasers," Proc. IEEE, vol. 58, pp. 1342-1357, September 1970.

[44] A. Yariv, "Internal modulation in multimode laser oscillators," J. Appl. Phys., vol. 36, pp. 388-391, February 1965.

[45] P. W. Smith, "Phase locking of laser modes by continuous cavity length variations," Appl. Phys. Lett., vol. 10, pp. 51-53, January 15, 1967.

[46] - "The self-pulsing laser oscillator," IEEE J. Quantum Electron., vol. QE-3, pp. 627-635, November 1967.

[47] A. A. Vuylsteke, "Theory of laser regeneration switching," J. Appl Phys., vol. 34, pp. 1615-1622, June 1963.

[48] W. H. Steier, "Coupling of high peak power pulses from $\mathrm{He}-\mathrm{Ne}$ lasers," Proc. IEEE (Letters), vol. 54, pp. 1604-1606, November 1966.

[49] R. B. Chesler and D. Maydan, "A calculation of Nd:YAlG cavity dumping" (to be published).

[50] R. W. Hellwarth, "Control of fluorescent pulsations," in Advances in Quantum Electronics, J. R. Singer, Ed. New York: Columbia University Press, 1961, pp. 334-341.

[51] F. J. McClung and R. W. Hellwarth, "Giant optical pulsations from ruby," J. Appl. Phys., vol. 33, pp. 828-829, March 1962.

[52] J. E. Geusic, M. L. Hensel, and R. G. Smith, "A repetitively $Q$. switched, continuously pumped YAlG:Nd laser," Appl. Phys. Lett., vol. 6, pp. 175-177, May 1965.

[53] E. J. Woodbury, "Five kilohertz repetition rate pulses YAIG:Nd laser," IEEE J. Quantum Electron., vol. QE-3, pp. 509-516, November 1967.

[54] V. Evtuhov and J. K. Neeland, "A continuously pumped repetitively $Q$-switched ruby laser and applications to frequency conversion experiments," IEEE J. Quantum Electron., vol. QE-5, pp. 207-209, April 1969

[55] R. B. Chesler, M. A. Karr, and J. E. Geusic, "A practical, highrepetition rate $Q$-switched $\mathrm{Nd}$ :YAlG laser," IEEE J. Quantum Electron., vol. QE-5, p. 345, June 1969.

[56] P. K. Cheo and H. G. Cooper, "Ultraviolet ion laser transitions between $2300 \AA$," J. Appl. Phys., vol. 36, pp. 1862-1865, January 1965.

[57] H. Steffen, J. Steffen, J. F. Moser, and F. K. Kneubuhl, "Comments on a new laser emission at $0.774 \mathrm{~mm}$ wavelength from ICN," Phys. Lett., vol. 21, pp. $425-426,1966$.

[58] F. Horrigan, R. Rudko, and D. Wilson, "High-power $10.6 \mu$ windows," presented at the 1968 Internatl. Quantum Electron. Conf., Miami, Fla

[59] For example, the Spectra-Physics Model $119 \mathrm{He}-\mathrm{Ne}$ laser.

[60] D. A. Leonard, "Saturation of the molecular nitrogen second positive laser transition," Appl. Phys. Lett., vol. 7, pp. 4-6, July 1965.

[61] A. J. DeMaria, "Electrically excited $\mathrm{CO}_{2}$ lasers," Bull. Amer. Phys. Soc., vol. 15, p. 563, April 1970.

[62] P. Rabinowitz, S. Jacobs, and G. Goulds, "Continuous optically pumped Cs laser," Appl. Optics, vol. 1, pp. 513-516, 1962.

[63] $\mathrm{I}$. Wieder, "Flame pumping and infrared maser action in $\mathrm{CO}_{2}$," Phys. Lett., vol. 13, pp. 759-760, June 19, 1967. 
[64] D. J. Spencer, H. Mirels, T. A. Jacobs, and R. W. F. Gross, "Preliminary performance of a CW chemical laser," Appl. Phys. Lett., vol. 16, pp. 235-237, March 1970.

[65] T. A. Cool and R. R. Stephens, "Chemical laser by fluid mixing," J. Chem. Phys., vol. 51, pp. 5175-5176,1969.

[66] E. T. Gerry, "Gas-dynamic $\mathrm{CO}_{2}$ lasers," Bull. Amer. Phys. Soc., vol. 15, p. 563, April 1970.

[67] J. V. V. Kasper and G. C. Pimentel, "Atomic iodine photodissociation laser," Appl. Phys. Lett., vol. 5, pp. 231-233, December 1, 1964.

[68] T. F. Deutsch, "New infrared laser transitions in $\mathrm{HCl}, \mathrm{HBr}, \mathrm{DCl}$, and DBr," IEEE J. Quantum Electron., vol. QE-3, pp. 419-421, October 1967.

[69] D. L. Perry, "Low-loss multilayer dielectric mirrors," Appl. Optics, vol. 4 , p. $987,1965$.

[70] A. D. White and E. I. Gordon, "Excitation mechanisms and current dependence of population inversion in He-Ne lasers," Appl. Phys. Lett., vol. 3, p. 197, 1963.

[71] D. A. Crane, Hughes Electron Dynamics Division, Malibu, Calif., private communication.

[72] W. B. Bridges and W. P. Kolb, "The first space-qualified laser," Laser Focus, vol. 5, pp. 39-42, October 1969.

[73] W. B. Bridges and A. S. Halsted, "Gaseous ion laser research," Hughes Res. Lab., Malibu, Calif., Tech. Rep. AFAL-TR-67-89, DDC, ASTIA Doc., AD814897, May 1967.

[74] E. I. Gordon, E. F. Labuda, and W. B. Bridges, "Continuous visible laser action, in singly ionized argon, krypton and xenon," Appl. Phys. Lett., vol. 4, p. 178, 1964.

[75] W. B. Bridges, A. S. Halsted, and G. N. Mercer, Hughes Res. Lab., Malibu, Calif. (unpublished).

[76] E. F. Labuda, E. I. Gordon, and R. C. Miller, "Continuous-duty argon ion lasers," IEEE J. Quantum Electron., vol. QE-1, pp. 273279 , September 1965.

[77] K. G. Hernqvist and J. R. Fendley, Jr., "Construction of long life argon laser," IEEE J. Quantum Electron., vol. QE-3, pp. 66-72, February 1967.

[78] R. A. Paananen, A. Adams, Jr., and D. T. Wilson, "Characteristics of metal wall ionized argon lasers,” I965 NEREM Rec., pp. 238-239.

[79] J. D. Rigden, "A metallic plasma tube for ion lasers," IEEE J. Quantum Electron. (Correspondence), vol. QE-1, p. 221, August 1965.

[80] W. B. Bridges and G. N. Mercer, "Ultraviolet ion laser," Hughes Res. Lab., Malibu, Calif., Tech. Rept. ECOM-0229-F, October 1969.

[81] C. K. N. Patel, W. L. Faust, and R. L. McFarlane, "CW laser action on rotational transitions of the $\Sigma_{u}^{+}-\Sigma_{0}^{+}$vibrational band of $\mathrm{CO}_{2}$," Bull. Amer. Phys. Soc., vol. 9, p. 500, 1964.

[82] N. Legay- Sommaire, L. Henry, and F. Legay, "Réalization d'un laser utilisant l'énergie de vibration des gaz excités par l'azote activé (CO, $\mathrm{CO}_{2}$, et $\mathrm{N}_{2} \mathrm{O}$ ), Compt. Rend., vol. 260, pp. 3339, 1965.

[83] C. K. N. Patel, "Vibration energy transfer, an efficient means of selective excitation," presented at the 1965 Conf. on Physics of Quantum Electron., San Juan, Puerto Rico.

[84] M. R. Smith and D. C. Forster, "High-power laser amplifier at $10.6 \mu$," presented at the 1968 Internatl. Quantum Electron. Conf., Miami, Fla.

[85] W. B. Tiffany, R. Targ, and J. D. Foster, "Kilowatt $\mathrm{CO}_{2}$ gas transport laser," Appl. Phys. Lett., vol. 15, pp. 91-93, August 1, 1969.

[86] W. J. Witteman, "Increasing continuous laser action on $\mathrm{CO}_{2}$ rotational vibrational transitions through selective depopulation of the lower laser level by means of water vapor," Phys. Lett., vol. 13, p. $125,1965$.

[87] P. O. Clark and J. Y. Wada, "The influence of xenon on sealed-off $\mathrm{CO}_{2}$ lasers," IEEE J. Quantum Electron., vol. QE-4, pp. 263-266, May 1968.

[88] F. E. Goodwin, Hughes Research Laboratories, Malibu, Calif., private communication.

[89] F. E. Goodwin, private communication.

[90] M. R. Johnson and N. Holonyak, Jr., "Optically pumped thinplatelet semiconductor lasers," J. Appl. Phys., vol. 39, pp. 39773985 , July 1968.

[91] N. G. Basov, A. Z. Grasiuk, V. F. Efimkov, I. G. Zubarev, V. A. Katulin, and J. M. Popov, "Semiconductor lasers using optical pumping," J. Phys. Soc. Jap. (suppl)., vol. 21, pp. 277-282, 1966.

[92] P. D. Southgate, "Stimulated emission from bulk field-ionized GaAs," IEEE J. Quantum Electron., vol. QE-4, pp. 179-185, April 1968.

[93] N. G. Basov, O. V. Bogdankevich, A. N. Pechenov, A. S. Nasibov, and K. P. Fedoseev, "Semiconductor laser with external resonator,"
Sov. Phys.-JETP, vol. 28, pp. 900-903, May 1969.

[94] W. F. Kosonocky, R. Cornely, and W. Hegyi, "Multilayer GaAs injection laser," IEEE J. Quantum Electron., vol. QE-4, pp. 176-179, April 1968.

[95] W. E. Engeler and M. Garfinkel, "Characteristics of a continuous high-power GaAs junction laser," J. Appl. Phys., vol. 35, pp. 1734 1741, June 1964.

[96] J. I. Pankove, "Temperature dependence of emission efficiency and lasing threshold in laser diodes," IEEE J. Quantum Electron., vol. QE-4, pp. 119-125, April 1968.

[97] H. Kressel and H. Mierop, "Catastrophic degradation in GaAs injection lasers," J. Appl. Phys., vol. 38, pp. 5419-5421, December 1967.

[98] M. Ettenberg, private communication.

[99] H. Kressel and N. E. Byer, "Physical basis of noncatastrophic degradation in GaAs injection lasers," Proc. IEEE, vol. 57, pp. 2533, January 1969.

[100] J. E. Ripper, "Comments on the reliability of GaAs stripe geometry junction lasers," IEEE J. Quantum Electron., vol. QE-6, pp. 372373, June 1970.

[101] J. C. Dyment and L. A. D'Asaro, "Continuous operation of GaAs junction lasers on diamond heat sink at $200^{\circ} \mathrm{K}$," Appl. Phys. Lett., vol. 11, pp. 292-294, November 1967.

[102] W. F. Kosonocky and R. Cornely, "GaAs laser amplifiers," IEEE J. Quantum Electron., vol. QE-4, pp. 125-131, April 1968.

[103] T. L. Paoli and J. E. Ripper, "Coupled longitudinal mode pulsing in semiconductor lasers," Phys. Rev. Lett., vol. 22, pp. 1085-1088, May 1969.

[104] L. A. D'Asaro, J. M. Cherlow, and T. L. Paoli, "Continuous microwave oscillations in GaAs junction lasers," IEEE J. Quantum Electron., vol. QE-4, pp. 164-167, April 1968.

[105] J. Feinlieb, S. Graves, W. Paul, and R. Zallen, "Effect of pressure on the spontaneous and stimulated emission from GaAs," Phys. Ret., vol. 131, pp. 2070-2078, September 1963.

[106] a) F. M. Ryan and R. C. Miller, "The effect of uniaxial strain on the threshold current and output of GaAs lasers," Appl. Phys. Lett., vol. 3, pp. 162-163, November 1963.

b) D. Meyerhofer and R. Braunstein, "Frequency tuning of GaAs laser diodes by uniaxial stress," Appl. Phys. Lett., vol. 3, pp. 171172, November 1963.

[107] J. M. Besson, J. F. Butler, A. R. Calawa, W. Paul, and R. H. Rediker, "Pressure-tuned PbSe diode laser," Appl. Phys. Lett., vol. 7, pp. 206-208, October 1965.

[108] R. J. Phelan, A. R. Calawa, R. H. Rediker, R. J. Keyes, and B. Lax, "Infrared InSb laser diode in high magnetic fields," Appl. Phys. Lett., vol. 3, pp. 143-145, November 1963.

[109] G. C. Dousmanis and D. L. Staebler, "Large wavelength changes with cavity $Q$ in injection lasers," $J$. Appl. Phys., vol. 37, pp. 1180 2278, May 1966.

[110] G. E. Fenner, "Internal frequency modulation of GaAs junction lasers by changing the index of refraction through electron injection," Appl. Phys. Lett., vol. 5, pp. 198-199, November 1964.

[111] S. Takamiya, F. Kitasawa, and J. Nishizawa, "Amplitude modulation of diode laser light in millimeter-wave region," Proc. IEEE (Letters), vol. 56, pp. 135-136, January 1968.

[112] T. L. Paoli and J. E. Ripper, "Direct modulation of semiconductor lasers," Proc. IEEE, this issue, pp. 1457-1465.

[113] H. Kressel and H. Nelson, "Close-confinement GaAs p-n junction lasers with reduced optical loss at room temperature," $R C A R e v$., vol. 30, pp. 106-113, March 1969.

[114] I. Hayashi, M. B. Panish, and P. Foy, "A low-threshold roomtemperature injection laser," IEEE J. Quantum Electron., vol. QE-5, pp. 211-212, April 1969.

[115] Jh. I. Alferov, V. M. Andreev, V. I. Korolkov, E. L. Portnoi, and D. N. Tretyakov, "Coherent radiation of epitaxial heterojunction structures in the AlAs-GaAs system," Sov. Phys.-Semiconduct., vol. 2, pp. 1289-1291, April 1969.

[116] L. F. Johnson, G. D. Boyd, K. Nassau, and R. R. Soden, "Continuous operation of a CaWO $4: \mathrm{Nd}^{3+}$ optical maser," Proc. IRE (Correspondence), vol. 50, p. 213, February 1962.

[117] V. Evtuhov and J. K. Neeland, "Continuous operation of a ruby laser at room temperature," Appl. Phys. Lett., vol. 6, pp. 75-76, February 15, 1965.

[118] D. Röss, "Room temperature ruby CW laser," Microwaves, vol. 3, p. 29, April 1965.

[119] C. G. Young, "Continuous glass laser," Appl. Phys. Lett., vol. 2, pp. 151-152, April 15, 1963 .

[120] a) J. E. Geusic, H. M. Marcos, and L. G. Van Uitert, "Laser 
oscillations in Nd-doped yttrium aluminum, yttrium gallium and gadolinium garnets," Appl. Phys. Lett., vol. 4, pp. 182-184, May 15, 1964.

b) J. E. Geusic, "The YAIG: Nd laser," Solid-state maser research (optical) Final Rept., U. S. Army Electron. Material Agency, contract DA-36-039AMC-02333E, August 30, 1965.

[121] R. C. Duncan, Jr., "Continuous room temperature $\mathrm{Nd}^{3+}: \mathrm{CaMoO}_{4}$ laser," J. Appl. Phys., vol. 36, pp. 874-875, March 1965.

[122] R. C. Ohlmann, K. B. Steinbruegge, and R. Mazelsky, "Spectroscopic and laser characteristics of neodymium-doped calcium fluoroapatite," Appl. Opt., vol. 7, pp. 905-914, May 1968.

[123] a) Kh. S. Bagdasarov and A. A. Kaminskii, "YAlO ${ }_{3}$ with $\mathrm{TR}^{3+}$ ion impurity as an active laser medium," JETP Lett., vol. 9, p. 303, May 5, 1969.

b) M. J. Weber, M. Bass, K. Andringa, R. R. Montchamp, and E. Comperchio, "Czochralski growth and properties of $\mathrm{YAlO}_{3}$ crystals," Appl. Phys. Lett., vol. 15, pp. 342-345, November 15, 1969.

[124] R. V. Alves, K. A. Wickersheim, and R. A. Buchanan, "Optical characteristics and laser performance of neodymium activated lanthanum oxysulfide," Bull. Amer. Phys. Soc., March 1970.

[125] W. A. Specht, Jr., J. K. Neeland, and V. Evtuhov, "Output spectra of Nd:YAG and ruby lasers and implications for laser linewidth determining mechanisms," IEEE J. Quantum Electron., vol. QE-2, pp. 537-541, September 1966.

[126] V. Evtuhov and J. K. Neeland, "Power output and efficiency of continuous ruby lasers," J. Appl. Phys., vol. 38, p. 4051, 1967.

[127] D. Röss, "Analysis of room temperature CW ruby lasers," IEEE J. Quantum Electron., vol. QE-2, pp. 208-214, August 1966.

[128] T. Kushida and J. E. Geusic, "Optical refrigeration in Nd-doped yttrium aluminum garnet," Phys. Rer. Lett., vol. 21, pp. 1172-1175, October 14, 1968.

[129] T. Kushida, H. M. Marcos, and J. E. Geusic, "Laser transition cross section and fluorescence branching ratio for $\mathrm{Nd}^{3+}$ in yttrium aluminum garnet," Phys. Rev., vol. 167, pp. 289-291, March 10, 1968.

[130] - "Laser transition crossections of lines other than $1.064 \mu$ in Nd:YAlG" (unpublished).

[131] R. G. Smith, "New room temperature CW laser transitions in YAlG: Nd," IEEE J. Quantum Electron. (Correspondence), vol. QE-4, pp. 505-506, August 1968.

[132] W. W. Benson and J. E. Geusic (unpublished).

[133] C. H. Church and I. I-iberman, "The spherical reflector for use in the optical pumping of lasers," Appl. Opt., vol. 6, pp. 1966-1968, November 1967.

[134] D. Röss, "CW and quasi-CW solid state lasers," Siemens Z., vol. 41, pp. 3-11, 1967.

[135] W. W. Benson, H. M. Marcos, and J. E. Geusic (unpublished).

[136] T. B. Read, "The CW pumping of YAlG: $\mathrm{Nd}^{3+}$ by water-cooled krypton arcs," Appl. Phys. Lett., vol. 9, pp. 342-344, November 1, 1966.

[137] I. Liberman and R. L. Grassel, "A comparison of lamps for use in high continuous power Nd:YAlG lasers," Appl. Opt., vol. 8, pp. 1875-1878, September 1969.

[138] I. Liberman, D. A. Larson, and C. H. Church, "Efficient Nd:YAG CW lasers using alkali additive lamps," IEEE J. Quantum Electron., vol. QE-5, pp. 238-241, May 1969.

[139] E. G. Erickson, "Holobeam reports 760 watts $\mathrm{CW}$ from a segmented Nd:YAlG system," Laser Focus, p. 16, April 1970.

[140] W. W. Benson and J. E. Geusic (unpublished).

[141] I. Liberman, "High-power Nd: YAG continuous laser" (Abstract), IEEE J. Quantum Electron., vol. QE-5, p. 345, June 1969.

[142] L. M. Osterink and J. D. Foster, "Efficient, high-power Nd:YAG laser characteristics" (Abstract), IEEE J. Quantum Electron., vol. QE-5, pp. 344-345, June 1969.

[143] W. Koechner, "YAlG challenges carbon dioxide in high $\mathrm{CW}$ power," Laser Focus, pp. 29-34, September 1969.

[144] J. D. Foster and L. M. Osterink, "Birefringence in continuously pumped Nd:YAIG rods" (to be published).

[145] J. D. Foster and L. M. Osterink, "Index of refraction and expansion coefficients of Nd:YAlG," Appl. Opt., vol. 7, pp. 2428-2429,
December 1968.

[146] R. T. Daly and R. A. Kaplan, "Thermally induced birefringence in a pumped laser rod," Quantronix Corporation (unpublished).

[147] W. Koechner and D. K. Rice, "Effect of birefringence on the performance of linearly polarized Nd:YAlG lasers" (to be published).

[148] L. M. Osterink and J. D. Foster, "Experimental compensation of thermally induced stress birefringence in solid state laser rods," 1969 Proc. Internatl. Electron Device Meeting, p. 70.

[149] J. E. Geusic and H. M. Marcos (unpublished).

[150] R. B. Chesler, "A stabilizing sleeve for the YAlG laser" (to be published).

[151] J. E. Geusic, "Advances in CW solid state lasers," 1966 NEREM Rec, p. 192.

[152] J. E. Geusic, "The continuous Nd:YAG laser" (Abstract), IEEE J. Quantum Electron., vol. QE-2, p. xvii, April 1966.

[153] H. G. Danielmeyer and W. G. Nilsen, "Spontaneous single frequency output from a spatially homogeneous Nd:YAlG laser," Appl. Phys. Lett., vol. 16, pp. 124-126, February 1970.

[154] L. M. Osterink and J. D. Foster, "A mode-locked Nd:YAlG laser," J. Appl. Phys., vol. 39, pp. 4163-4165, August 1968.

[155] G. W. Hong and J. R. Whinnery, "Switching of phase-locked states in the intracavity phase-modulated He-Ne laser," IEEE J. Quantum Electron., vol. QE-5, pp. 367-376, July 1969.

[156] D. L. Lyon and T. S. Kinsel, "Transitions between mode-locked states in intracavity phase-modulated lasers," Appl. Phys. Lett., vol. 16, pp. 89-91, February 1970.

[157] T. S. Kinsel, J. E. Geusic, H. Seidel, and R. G. Smith, “A stabilized mode-locked Nd: YAlG laser source" (Abstract), IEEE J. Quantum Electron., vol. QE-5, p. 326, June 1969.

[158] R. B. Chesler, M. A. Karr, and J. E. Geusic, "A practical highrepetition rate $Q$-switched Nd:YAlG laser" (to be published).

[159] D. Maydan and R. B. Chesler, " $Q$-switching and cavity dumping of $\mathrm{Nd}$ : YAlG lasers" (to be published).

[160] a) J. E. Geusic, H. J. Levinstein, J. J. Rubin, S. Singh, and L. G. Van Uitert, "The nonlinear optical properties of $\mathrm{Ba}_{2} \mathrm{NaNb}_{5} \mathrm{O}_{15}$," Appl. Phys. Lett., vol. 11, pp. 269-271, November 1967.

b) S. Singh, D. A. Draegert, and J. E. Geusic, "Optical and ferroelectric properties of barium sodium niobate," Phys. Rev. (to be published).

[161] J. E. Geusic and H. M. Marcos (unpublished).

[162] M. A. Karr, Bell Telephone Laboratories, Inc., private communication.

[163] R. B. Allen and S. J. Scalise, "Continuous operation of a YAlG: Nd laser by injection pumping," Appl. Phys. Lett., vol. 14, pp. 49-51, March 1969.

[164] F. W. Ostermayer, Bell Télephone Laboratories, Inc., private communication.

[165] B. Reed, Texas Instruments, Inc., private communication.

[166] R. G. Smith, J. E. Geusic, H. J. Levinstein, J. J. Rubin, S. Singh, and L. G. Van Uitert, "Continuous optical parametric oscillation in $\mathrm{Ba}_{2} \mathrm{NaNb}_{5} \mathrm{O}_{15}$," Appl. Phys. Lett., vol. 9, p. 308, May 1968.

[167] R. L. Byer, M. K. Oshman, J. F. Young, and S. E. Harris, "Visible CW parametric oscillator," Appl. Phys. Lett., vol. 13, p. 109, August 1968.

[168] R. G. Smith, Bell Telephone Laboratories, Inc., private communication.

[169] J. E. Bjorkholm, "Efficient optical parametric oscillation using doubly and singly resonant cavities," Appl. Phys. Lett., vol. 13, pp. 53-56, July 1968 .

[170] J. E. Bjorkholm, "Some spectral properties of doubly and singly resonant pulsed optical parametric oscillators," Appl. Phys. Lett., vol. 13, pp. 399-401, December 1960.

[171] L. B. Kreuzer, "Single mode oscillation of a pulsed singly resonant optical parametric oscillator," Appl. Phys. Lett., vol. 15, pp. 263265, October 1969.

[172] J. Falk and J. E. Murray, "Single cavity noncollinear optical parametric oscillation," Appl. Phys. Lett., vol. 14, pp. 245-247, April 1969.

[173] F. S. Chen, "Modulators for optical communication," Proc. IEEE, this issue, pp. 1440-1457.

[174] H. Melchior, M. B. Fisher, and F. Arams. "Photodetectors for optical communication systems," Proc. IEEE, this issue, pp. 14661486. 


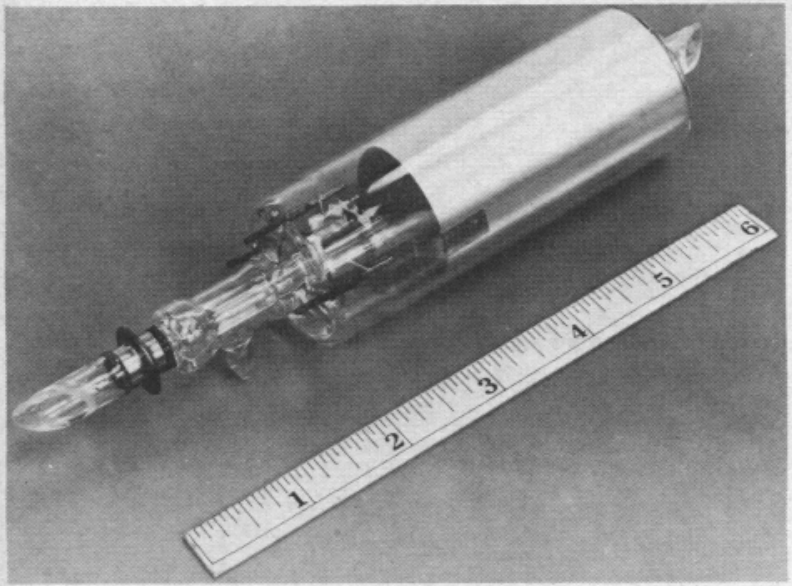

Fig. 6. Typical small $(0.5 \mathrm{~mW})$ external-mirror $\mathrm{He}-\mathrm{Ne}$ laser.

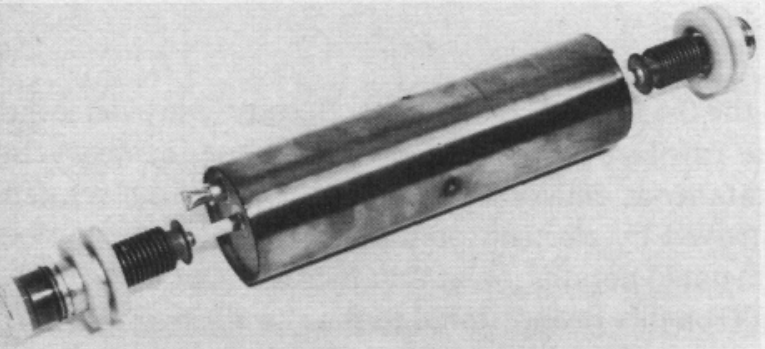

(a)

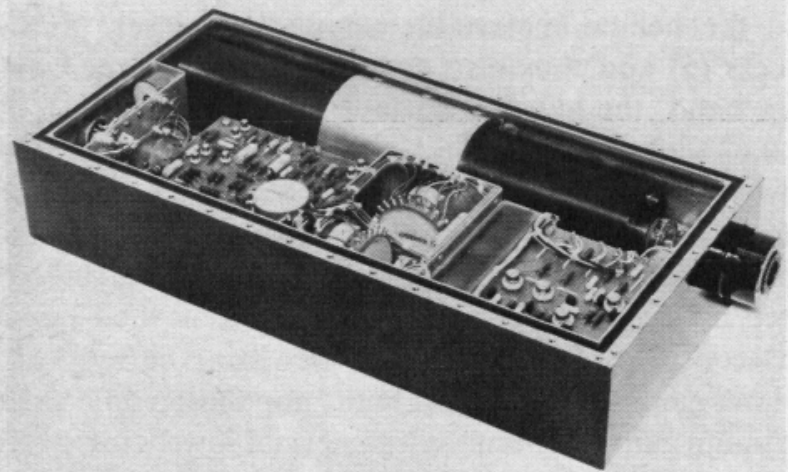

(b)

Fig. 7. (a) Metal-ceramic internal-mirror $\mathrm{He}-\mathrm{Ne}$ lasers for space-qualfied systems. (b) Integrated space-qualified $\mathrm{He}-\mathrm{Ne}$ laser package (5 $\mathrm{mW}$ ) with all solid-state power conditioning system. Prime power requirements are 30 watts at $24-32$ volt dc. 


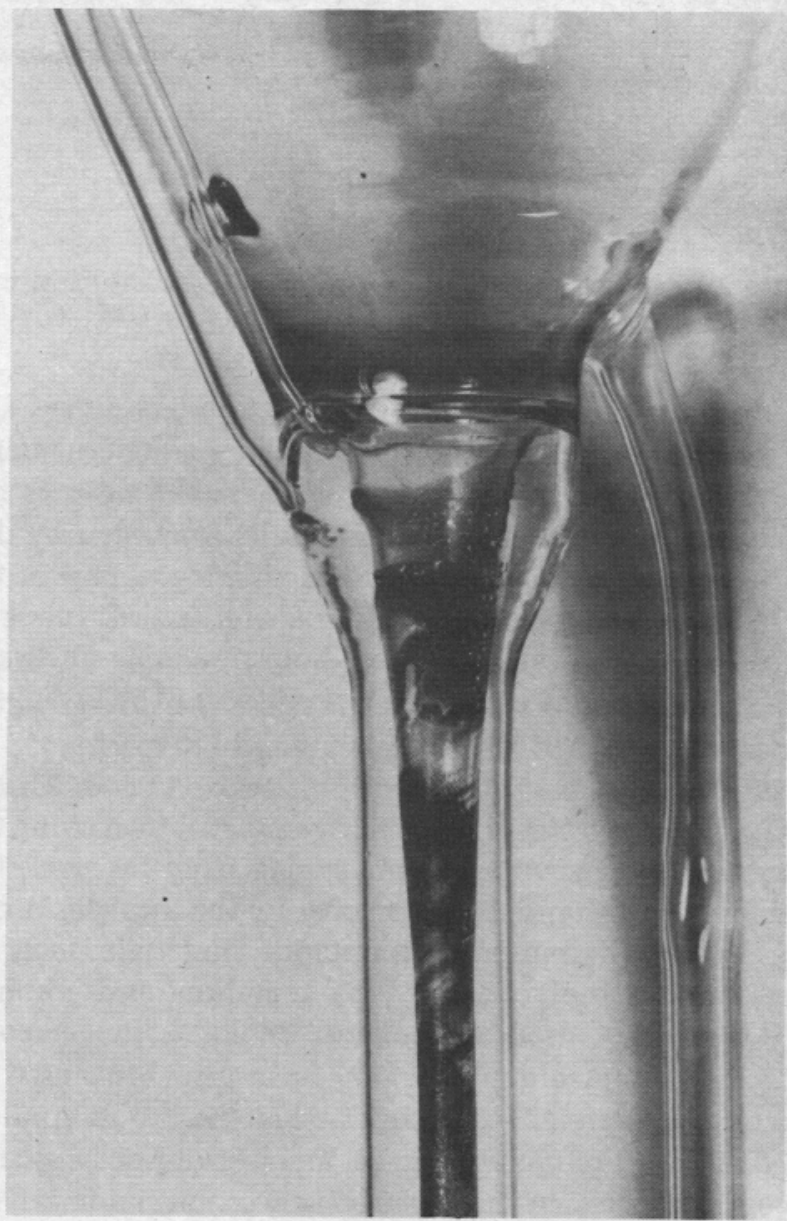

Fig. 8. Cathode throat region of typical $\mathrm{CW}$ ion laser silica discharge tube after 100 hours of operation. Discoloration is silicon or silicon monoxide from ion-decomposed $\mathrm{SiO}_{2}$. 


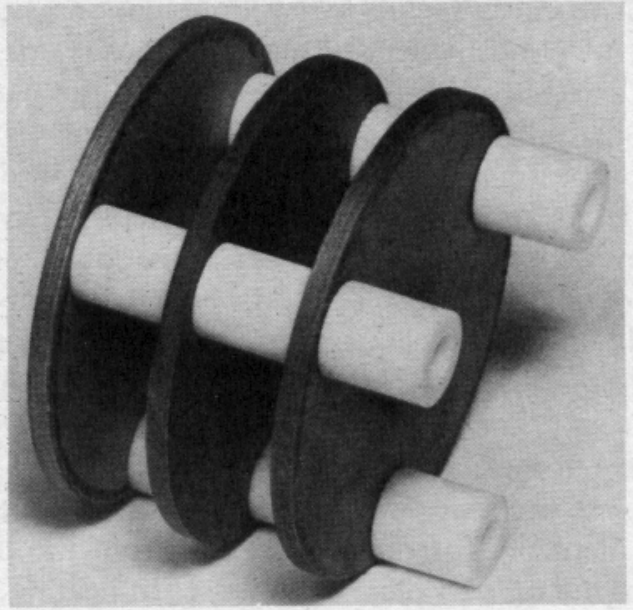

Fig. 10. Stacked tungsten disks used to form radiation-cooled bore of a high-power ultraviolet ion laser. 


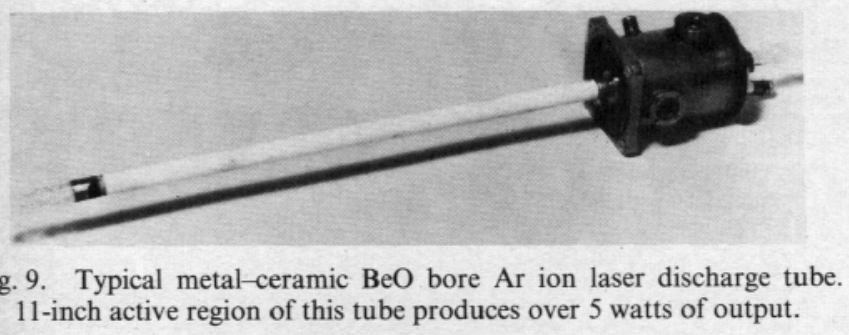

Fig. 9. Typical metal-ceramic $\mathrm{BeO}$ bore $\mathrm{Ar}$ ion laser discharge tube. 11-inch active region of this tube produces over 5 watts of output. 


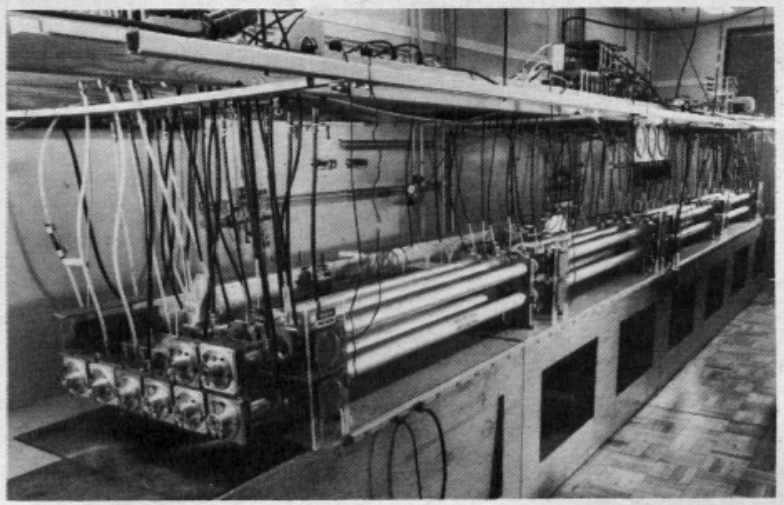

Fig. 11. Early breadboard version of $1.5-\mathrm{kW}$ average power, $15-\mathrm{kW}$ peak power MOPA $\mathrm{CO}_{2}$ laser system. 


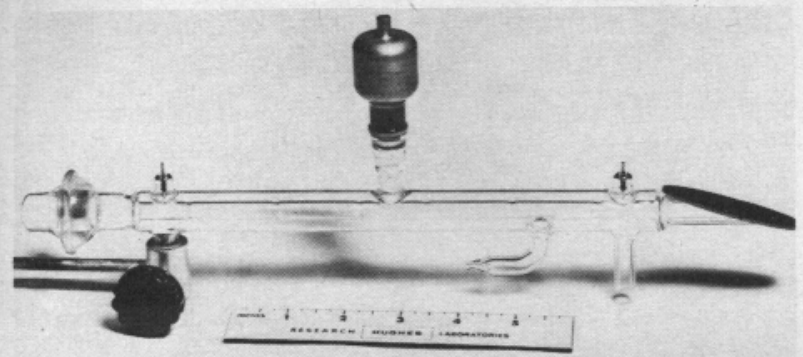

Fig. 12. Small glass sealed-off $\mathrm{CO}_{2}$ laser used in life test studies. 


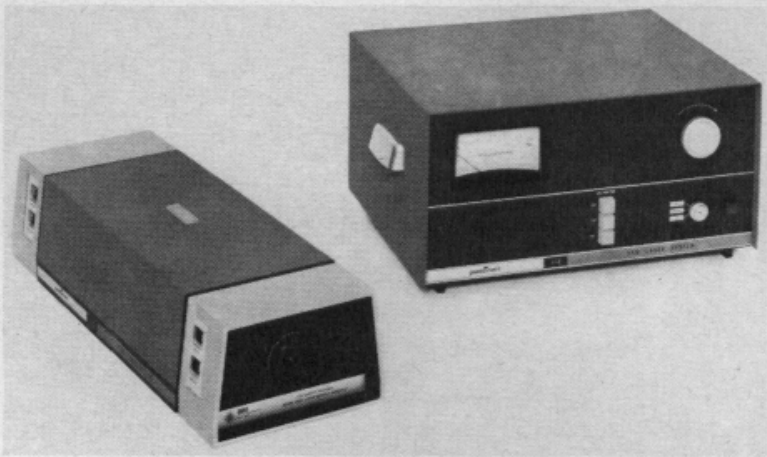

Fig. 18. Commercial, W-I pumped Nd:YAlG laser which has rated output of $\sim 10$ watts. Lamp power supply and closed cycle water cooling systems are shown on the right. 
DIODE DOME

( EMITTING ELEMENT

YAIG :

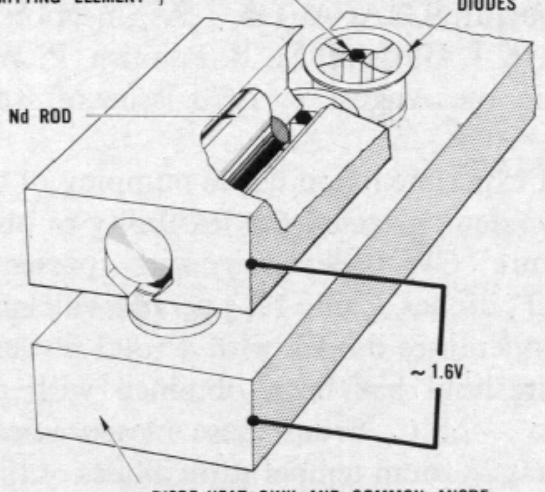

DIODE HEAT SINK AND COMMON ANODE

Fig. 20. Diagram of a diode-pumped Nd:YAlG laser. Diodes depicted are incoherent domed emitters such as GaAsP.

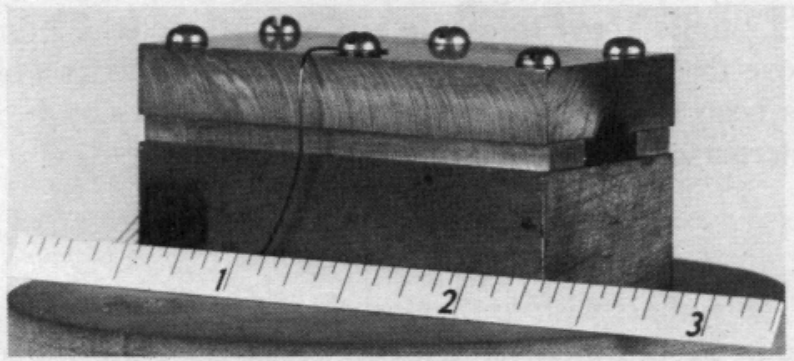

Fig. 21. Working model of diode-pumped Nd:YAlG laser. This model uses diodes which have to be cooled to $77^{\circ} \mathrm{K}$ in order to shift their spectral output into the desired $0.81-\mu$ pump band region.

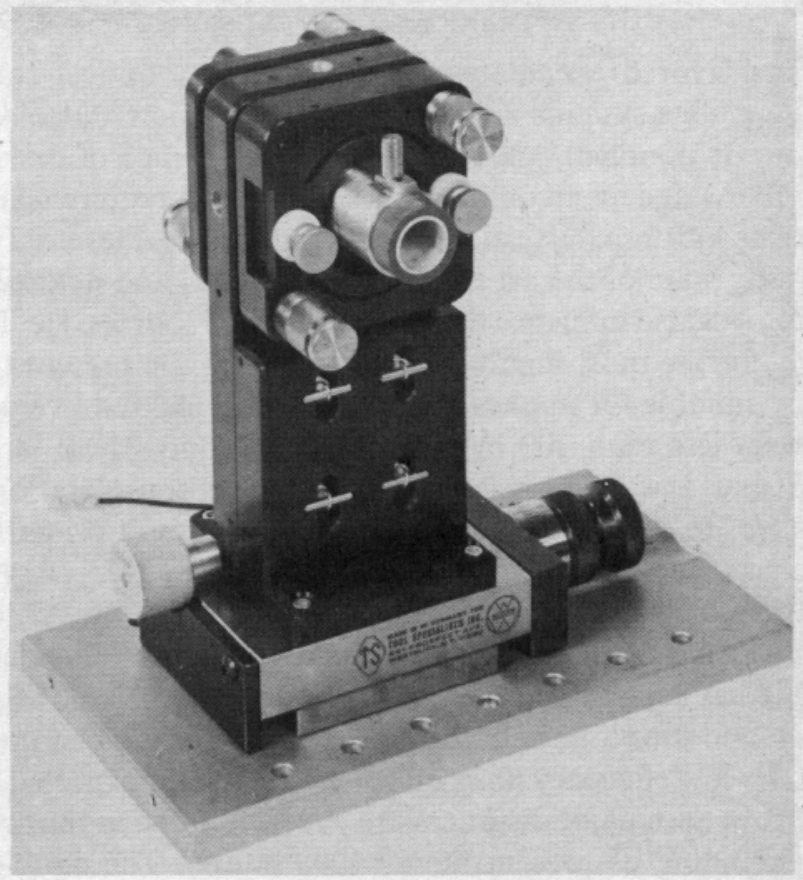

Fig. 22. Doubly resonant $\mathrm{CW}$ parametric oscillator.

idler. In the oscillator of Smith et al. [166], the nonlinear material was $\mathrm{Ba}_{2} \mathrm{NaNb}_{5} \mathrm{O}_{15}[160]$ and the pump was a $0.532-\mu$ doubled Nd:YAlG source. A threshold of 45 $\mathrm{mW}$, an output power of $3 \mathrm{~mW}$, and a tuning range from 0.98 to $1.16 \mu$ were observed. The outputs from these early continuous parametric oscillators had severe amplitude 